\title{
SOME REMARKS ON CONTACT VARIATIONS IN THE FIRST HEISENBERG GROUP
}

\author{
Sebastiano Golo \\ University of Trento, Department of Mathematics \\ Via Sommarive 14, 38123 Povo, Italy; s.nicolussigolo@unitn.it
}

\begin{abstract}
We show that in the first sub-Riemannian Heisenberg group there are intrinsic graphs of smooth functions that are both critical and stable points of the sub-Riemannian perimeter under compactly supported variations of contact diffeomorphisms, despite the fact that they are not area-minimizing surfaces. In particular, we show that if $f: \mathbf{R}^{2} \rightarrow \mathbf{R}$ is a $\mathscr{C}^{1}$-intrinsic function, and $\nabla^{f} \nabla^{f} f=0$, then the first contact variation of the sub-Riemannian area of its intrinsic graph is zero and the second contact variation is positive.
\end{abstract}

\section{Introduction}

We want to address some new features of the sub-Riemannian perimeter in the Heisenberg group. The notion of sub-Riemannian perimeter in the Heisenberg group, the so-called intrinsic perimeter, has been enstablished as a direct and natural extension from the Euclidean perimeter in $\mathbf{R}^{n}$. However, in many aspects, there are fundamental differences that lead to new open questions $[6,7,18,10,16]$.

Before a detailed explanation, let us introduce some basic notions and notations we need in this introduction. The (first) Heisenberg group $\mathbf{H}$ is a three dimensional Lie group diffeomorphic to $\mathbf{R}^{3}$. However, when endowed with a left-invariant subRiemannian distance, it becomes a metric space with Hausdorff dimension equal to four; see [3].

By standard methods of Geometric Measure Theory, one defines the intrinsic perimeter $P(E ; \Omega)$ of a measurable set $E \subset \mathbf{H}$ in an open set $\Omega \subset \mathbf{H}$. We will denote it also by $\mathscr{A}(\partial E \cap \Omega)$.

Regular surfaces are topological surfaces in $\mathbf{H}$ that admit a continuously varying tangent plane and they play an important role in the theory of sets with finite intrinsic perimeter. They are the sub-Riemannian counterpart of smooth hypersurfaces in $\mathbf{R}^{n}$. Regular surfaces are locally graphs of so-called $\mathscr{C}^{1}$-intrinsic functions $\mathbf{R}^{2} \rightarrow \mathbf{R}$. We will focus on these functions and their graphs.

The space of all $\mathscr{C}^{1}$-intrinsic functions will be denoted by $\mathscr{C}_{\mathbf{W}}^{1}$ and the graph of $f: \mathbf{R}^{2} \rightarrow \mathbf{R}$ by $\Gamma_{f} \subset \mathbf{H}$. It is well known that $f \in \mathscr{C}_{\mathbf{W}}^{1}$ if and only if $f \in \mathscr{C}^{0}\left(\mathbf{R}^{2}\right)$ and the distributional derivative

$$
\nabla^{f} f=\partial_{\eta} f+\frac{1}{2} \partial_{\tau}\left(f^{2}\right)
$$

https://doi.org/10.5186/aasfm.2018.4312

2010 Mathematics Subject Classification: Primary 53C17, 49Q20.

Key words: Sub-Riemannian geometry, sub-Riemannian perimeter, Heisenberg group, Bernstein's problem, contact diffeomorphisms.

The author has been supported by the People Programme (Marie Curie Actions) of the European Union's Seventh Framework Programme FP7/2007-2013/ under REA grant agreement n. 607643, and by the Department of Mathematics and Statistics of the University of Jyväskylä, Finland. 
is continuous, where we denote by $(\eta, \tau)$ the coordinates on $\mathbf{R}^{2}$; see $[1,18]$. If $\omega \subset \mathbf{R}^{2}$, the intrinsic area of $\Gamma_{f}$ above $\omega$ is

$$
\mathscr{A}\left(\Gamma_{f} \cap \Omega_{\omega}\right)=\int_{\omega} \sqrt{1+\left(\nabla^{f} f\right)^{2}} \mathrm{~d} \eta \mathrm{d} \tau,
$$

where $\Omega_{\omega}=\{(0, \eta, \tau) *(\xi, 0,0):(\eta, \tau) \in \omega, \xi \in \mathbf{R}\}$, with $*$ denoting the group operation in $\mathbf{H}$.

An important open problem concerning $\mathscr{C}_{\mathbf{W}}^{1}$ is Bernstein's problem: If the graph $\Gamma_{f}$ of $f \in \mathscr{C}_{\mathbf{W}}^{1}$ is a locally minimizer of the intrinsic area, is $\Gamma_{f}$ a plane? See Section 2.4 for a precise statement and $[4,2,17,9]$ for further reading.

In the study of perimeter minimizers in $\mathbf{H}$, we identify three main issues that mark the gap from the Euclidean theory. First, the map $f \mapsto \nabla^{f} f$ is a nonlinear operator. Such non-linearity reflects on the fact that basic function spaces like $\mathscr{C}_{\mathbf{W}}^{1}$ itself, or the space of functions with bounded intrinsic variation, are not vector spaces. See Remark 2.3 for details.

Second, the area functional is not convex (say on $\mathscr{C}^{1}\left(\mathbf{R}^{2}\right)$ ). In particular, there are critical points that are not extremals, see [4]. In other words, a first variation condition

$$
\left.\frac{\mathrm{d}}{\mathrm{d} \epsilon}\right|_{\epsilon=0} \mathscr{A}\left(\Gamma_{f+\epsilon \phi} \cap\left(\Omega_{\omega}\right)\right)=0 \quad \forall \phi \in \mathscr{C}_{c}^{\infty}(\omega)
$$

does not characterize minimizers. However, if $f \in \mathscr{C}^{1}\left(\mathbf{R}^{2}\right)$, a second variation condition $\left.\frac{\mathrm{d}^{2}}{\mathrm{~d} \epsilon^{2}}\right|_{\epsilon=0} \mathscr{A}\left(\Gamma_{f+\epsilon \phi} \cap\left(\Omega_{\omega}\right)\right) \geq 0$ does, see [9].

Third, there are objects among sets of finite intrinsic perimeter with very low regularity, see Remark 2.3. The standard variational approach as in (1) fails when applied to these objects. More precisely, if $f \in \mathscr{C}_{\mathbf{W}}^{1}$, then $\mathscr{A}\left(\Gamma_{f+\epsilon \phi} \cap\left(\Omega_{\omega}\right)\right)$ may be $+\infty$ for all $\epsilon \neq 0$, all $\omega \subset \mathbf{R}^{2}$ open and all $\phi \in \mathscr{C}_{c}^{\infty}(\omega) \backslash\{0\}$. In another approach, one can consider smooth one-parameter families of diffeomorphisms $\Phi_{\epsilon}: \mathbf{H} \rightarrow \mathbf{H}$ with $\Phi_{0}=\mathrm{Id}$ and $\left\{\Phi_{\epsilon} \neq \mathrm{Id}\right\} \subset \subset \Omega$, and take variations of $\mathscr{A}\left(\Phi_{\epsilon}\left(\Gamma_{f}\right) \cap \Omega\right)$. However, it may happen again that $\mathscr{A}\left(\Phi_{\epsilon}\left(\Gamma_{f}\right) \cap \Omega\right)=+\infty$ for all $\epsilon \neq 0$.

After further considerations, one understands that we need to restrict the choice of $\Phi_{\epsilon}$ to contact diffeomorphisms, see Proposition 5.1. See also [3] and [12] for references on contact diffeomorphisms. In this setting, we address the question whether, despite this restriction, conditions on the first and second variations with contact diffeomorphisms can single out minimal graphs. Our answer is no:

Theorem 1.1. There is $f \in \mathscr{C}_{\mathbf{W}}^{1}$ such that, for all $\Omega \subset \mathbf{H}$ open and all smooth one-parameter families of contact diffeomorphisms $\Phi_{\epsilon}: \mathbf{H} \rightarrow \mathbf{H}$ with $\Phi_{0}=$ Id and $\left\{\Phi_{\epsilon}=\mathrm{Id}\right\} \subset \subset \Omega$, it holds

$$
\left.\frac{\mathrm{d}}{\mathrm{d} \epsilon}\right|_{\epsilon=0} \mathscr{A}\left(\Phi_{\epsilon}\left(\Gamma_{f}\right) \cap \Omega\right)=0 \quad \text { and }\left.\quad \frac{\mathrm{d}^{2}}{\mathrm{~d} \epsilon^{2}}\right|_{\epsilon=0} \mathscr{A}\left(\Phi_{\epsilon}\left(\Gamma_{f}\right) \cap \Omega\right) \geq 0,
$$

but $\Gamma_{f}$ is not an area-minimizing surface.

In fact, all smooth solutions to the equation $\nabla^{f}\left(\nabla^{f} f\right)=0$ are examples of the functions appearing in the theorem.

The proof of Theorem 1.1 is based on a "Lagrangian" approach to $\mathscr{C}_{\mathbf{W}}^{1}$. Indeed, a function $f \in \mathscr{C}_{\mathbf{W}}^{1}$ is uniquely characterized by the integral curves of the planar vector field $\nabla^{f}=\partial_{\eta}+f \partial_{\tau}$. We will thus take variations of $f$ via smooth one-parameter families of diffeomorphisms $\phi_{\epsilon}: \mathbf{R}^{2} \rightarrow \mathbf{R}^{2}$, i.e., by smoothly varying the integral 
curves of $\nabla^{f}$; see Section 4 . We will then prove that this approach is equivalent to the use of contact diffeomorphisms $\Phi_{\epsilon}: \mathbf{H} \rightarrow \mathbf{H}$; see Section 5 .

Finally, we will consider functions $f \in \mathscr{C}_{\mathbf{W}}^{1}$ that are solutions to the equation $\nabla^{f} \nabla^{f} f=0$ in a Lagrangian sense, that is, functions such that $\nabla^{f} f$ is constant along the integral curves of $\nabla^{f}$. We will characterize such functions as the ones for which the integral curves of $\nabla^{f}$ are parabolas, or, equivalentely, as the ones whose graph $\Gamma_{f}$ is ruled by horizontal straight lines. These functions are the ones appearing in Theorem 1.1.

The paper is organized as follows. Section 2 is devoted to the presentation of all main definitions. In the next Section 3, we study solutions to the equation $\nabla^{f} \nabla^{f} f=$ 0 . We construct a Lagrangian variation of a function $f \in \mathscr{C}_{\mathbf{W}}^{1}$ in Section 4 . In Section 5, we prove some basic properties of contact diffeomorphisms. Section 6 is devoted to the first contact variation and Section 7 to the second contact variation for functions $f \in \mathscr{C}_{\mathbf{W}}^{1}$. Finally, in Section 8 we prove our main theorem. An Appendix is added as a reference for a few equalities that are applied all over the paper.

The author thanks his advisor Francesco Serra Cassano for many fruitful discussions and Katrin Fässler for her comments and corrections on a draft of this paper.

\section{Preliminaries}

2.1. The Heisenberg group. The first Heisenberg group $\mathbf{H}$ is the connected, simply connected Lie group associated to the Heisenberg Lie algebra $\mathfrak{h}$. The Heisenberg Lie algebra $\mathfrak{h}$ is the only three-dimensional nilpotent Lie algebra that is not commutative. It can be proven that, for any two linearly independent vectors $A, B \in \mathfrak{h} \backslash[\mathfrak{h}, \mathfrak{h}]$, the triple $(A, B,[A, B])$ is a basis of $\mathfrak{h}$ and $[A,[A, B]]=$ $[B,[A, B]]=0$. The Heisenberg group has the structure of a stratified Lie group, i.e., $\mathfrak{h}=\operatorname{span}\{A, B\} \oplus \operatorname{span}\{[A, B]\}$, see $[14,13]$.

We then identify $\mathbf{H}=(\operatorname{span}\{A, B,[A, B]\}, *)$, where

$$
p * q:=p+q+\frac{1}{2}[p, q] .
$$

In the coordinates $(x, y, z)=x A+y B+z[A, B]$, which are the exponential coordinates of first kind, we have

$$
(a, b, c) *(x, y, z)=\left(a+x, b+y, c+z+\frac{1}{2}(a y-b x)\right) .
$$

The inverse is $(x, y, z)^{-1}=(-x,-y,-z)$.

The elements $A, B,[A, B] \in \mathfrak{h}$ induce a frame of left-invariant vector fields on $\mathbf{H}$ :

$$
X:=\partial_{x}-\frac{1}{2} y \partial_{z}, \quad Y:=\partial_{y}+\frac{1}{2} x \partial_{z}, \quad Z:=\partial_{z} .
$$

The horizontal subbundle is the vector bundle

$$
H:=\bigsqcup_{p \in \mathbf{H}} \operatorname{span}\{X(p), Y(p)\} \subset T \mathbf{H} .
$$

The maps $\delta_{\lambda}(x, y, z):=\left(\lambda x, \lambda y, \lambda^{2} z\right), \lambda>0$, are called dilations. They are group automorphisms of $\mathbf{H}$ and for all $\lambda, \mu>0$ it holds $\delta_{\lambda} \circ \delta_{\mu}=\delta_{\lambda \mu}$.

2.2. Intrinsic graphs and intrinsic differentials. A vertical plane is a plane containing the $z$-axis. Explicitly, for $\theta \in \mathbf{R}$,

$$
\mathbf{W}_{\theta}:=\{(\eta \sin \theta, \eta \cos \theta, \tau): \eta, \tau \in \mathbf{R}\} \subset \mathbf{H} .
$$


Vertical planes are the only 2-dimensional subgroups of $\mathbf{H}$ that are $\delta_{\lambda}$-homogeneous, i.e., $\delta_{\lambda}\left(\mathbf{W}_{\theta}\right)=\mathbf{W}_{\theta}$ for all $\lambda>0$.

The intrinsic $X$-graph (or simply intrinsic graph) of a function $f: \mathbf{R}^{2} \rightarrow \mathbf{R}$ is the $\operatorname{set}^{1}$

$$
\begin{aligned}
\Gamma_{f} & :=\left\{(0, \eta, \tau) *(f(\eta, \tau), 0,0): \eta, \tau \in \mathbf{R}^{2}\right\} \\
& =\left\{\left(f(\eta, \tau), \eta, \tau-\frac{1}{2} \eta f(\eta, \tau)\right): \eta, \tau \in \mathbf{R}^{2}\right\} .
\end{aligned}
$$

If one look at $f$ as a function $\mathbf{W}_{0} \rightarrow \operatorname{span}\{A\}$, then $\Gamma_{f}=\left\{p * f(p): p \in \mathbf{W}_{0}\right\}$. Left translations and dilations of an intrinsic graph are also intrinsic graphs. For $\alpha \in \mathbf{R}$, the vertical plane $\mathbf{W}_{\arctan (\alpha)}$ is the intrinsic graph of the function $f(\eta, \tau)=\alpha \eta$. We will use the map $\pi_{X}: \mathbf{H} \rightarrow \mathbf{R}^{2}, \pi_{X}(x, y, z)=\left(y, z+\frac{1}{2} x y\right)$. Note that $\pi_{X}(p * f(p))=p$.

For $\left(\eta_{0}, \tau_{0}\right) \in \mathbf{R}^{2}$ and $f: \mathbf{R}^{2} \rightarrow \mathbf{R}$ continuous, set $f_{0}:=f\left(\eta_{0}, \tau_{0}\right)$ and $p_{0}:=$ $\left(0, \eta_{0}, \tau_{0}\right) *\left(f_{0}, 0,0\right)=\left(f_{0}, \eta_{0}, \tau_{0}-\frac{1}{2} \eta_{0} f_{0}\right)$. We say that $f$ is intrinsically $\mathscr{C}^{1}$, or belonging to $\mathscr{C}_{\mathbf{W}}^{1}$, with differential $\psi: \mathbf{R}^{2} \rightarrow \mathbf{R}$, if $\delta_{\lambda}\left(p_{0}^{-1} \Gamma_{f}\right)$ converge to $\mathbf{W}_{\arctan \left(\psi\left(\eta_{0}, \tau_{0}\right)\right)}$ in the sense of the local Hausdorff convergence of sets as $\lambda \rightarrow \infty$, and the convergence is uniform on compact sets in $\left(\eta_{0}, \tau_{0}\right)$.

Notice that $\delta_{\lambda}\left(p_{0}^{-1} \Gamma_{f}\right)=\Gamma_{f_{\left(\eta_{0}, \tau_{0}\right) ; \lambda}}$, where

$$
f_{\left(\eta_{0}, \tau_{0}\right) ; \lambda}(\eta, \tau)=\lambda\left(-f_{0}+f\left(\eta_{0}+\frac{\eta}{\lambda}, \tau_{0}+f_{0} \frac{\eta}{\lambda}+\frac{\tau}{\lambda^{2}}\right)\right) .
$$

Therefore, $f$ belongs to $\mathscr{C}_{\mathbf{W}}^{1}$ with differential $\psi$ if and only if $f_{\left(\eta_{0}, \tau_{0}\right) ; \lambda}$ converge uniformly on compact sets to the function $(\eta, \tau) \mapsto \psi\left(\eta_{0}, \tau_{0}\right) \eta$, as $\lambda \rightarrow+\infty$, and the convergence is uniform on compact sets in $\left(\eta_{0}, \tau_{0}\right)$. Notice that $\psi$ has to be continuous.

The intrinsic gradient of a function $f: \mathbf{R}^{2} \rightarrow \mathbf{R}$ is the vector field on $\mathbf{R}^{2}$ defined as

$$
\nabla^{f}:=\partial_{\eta}+f \partial_{\tau}
$$

We can express the intrinsic differentiability in terms of the differentiability of $f$ along the integral curves of $\nabla^{f}$ : from [18, Theorem 4.95] we obtain the following characterisation, which justify the notation $\nabla^{f} f$ for the differential $\psi$ of $f \in \mathscr{C}_{\mathbf{W}}^{1}$.

Lemma 2.1. A continuous function $f: \mathbf{R}^{2} \rightarrow \mathbf{R}$ is in $\mathscr{C}_{\mathbf{W}}^{1}$ with differential $\psi$ if and only if for every $p \in \mathbf{R}^{2}$ there exists a $\mathscr{C}^{2}$-function $g_{p}: I \rightarrow \mathbf{R}$, where $I \subset \mathbf{R}$ is a neighbourhood of 0 , such that

$$
\begin{cases}g_{p}(0)=0, & \\ g_{p}^{\prime}(t)=f\left(p+\left(t, g_{p}(t)\right)\right) & \forall t \in I, \\ g_{p}^{\prime \prime}(t)=\psi\left(p+\left(t, g_{p}(t)\right)\right) & \forall t \in I .\end{cases}
$$

Note that $t \mapsto p+\left(t, g_{p}(t)\right)$ is an integral curve of $\nabla^{f}$ and that $g_{p}$ is not unique in general. Another interpretation of these curves will be useful:

Lemma 2.2. Let $f \in \mathscr{C}_{\mathbf{W}}^{1}$. A curve $\gamma: I \rightarrow \mathbf{R}^{2}$ of class $\mathscr{C}^{1}$, where $I \subset \mathbf{R}$ is an interval, is an integral curve of $\nabla^{f}$ if and only if the curve $t \mapsto \gamma(t) * f(\gamma(t)) \in \Gamma_{f}$ is a curve of class $\mathscr{C}^{1}$ tangent to the horizontal bundle $H$.

Remark 2.3. In [11] it has been shown that there exists $f \in \mathscr{C}_{\mathbf{W}}^{1}$ whose intrinsic graph $\Gamma_{f}$ has Euclidean Hausdorff dimension (seen as a subset of the Euclidean $\mathbf{R}^{3}$ )

\footnotetext{
${ }^{1}$ In a different choice of coordinates in $\mathbf{H}$, we can have $(0, \eta, \tau) *(f(\eta, \tau), 0,0)=(f(\eta, \tau), \eta, \tau)$. For instance, we will use these coordinates in Section 5.2.
} 
strictly larger than two. It is possible to prove, for example using Lemma 5.4, that $\Gamma_{f+1}$ does not have locally finite intrinsic perimeter and in particular $f+1 \notin \mathscr{C}_{\mathbf{W}}^{1}$. This shows that $\mathscr{C}_{\mathbf{W}}^{1}$ is not a vector space.

2.3. Smooth approximation. A sequence $\left\{f_{k}\right\}_{k \in \mathbf{N}} \subset \mathscr{C}_{\mathbf{W}}^{1}$ converges to $f$ in $\mathscr{C}_{\mathbf{W}}^{1}$ if $f_{k}$ and $\nabla^{f_{k}} f_{k}$ converge to $f$ and $\nabla^{f} f$ uniformly on compact sets. The following lemma has been proven in [1].

Lemma 2.4. If $f \in \mathscr{C}_{\mathbf{W}}^{1}$ then there is a sequence of functions $\left\{f_{k}\right\}_{k \in \mathbf{N}} \subset \mathscr{C}^{\infty}\left(\mathbf{R}^{2}\right)$ that converges to $f$ in $\mathscr{C}_{\mathbf{W}}^{1}$.

2.4. Perimeter and Bernstein's problem. The Lebesgue measure $\mathscr{L}^{3}$ in $\mathbf{R}^{3}$ is a Haar measure on $\mathbf{H}$ in the exponential coordinates introduced Section 2.1. Notice that for any measurable set $E \subset \mathbf{H}^{1}$ and any $\lambda>0$ it holds $\mathscr{L}^{3}\left(\delta_{\lambda}(E)\right)=\lambda^{4} \mathscr{L}^{3}(E)$.

Let $\langle\cdot, \cdot\rangle$ be the left-invariant scalar product on the subbundle $H$ such that $(X, Y)$ is an orthonormal frame and set $\|v\|:=\sqrt{\langle v, v\rangle}$ for $v \in H$. The sub-Riemannian perimeter of a measurable set $E \subset \mathbf{H}^{1}$ in an open set $\Omega$ is

$$
P(E ; \Omega):=\sup \left\{\int_{E} \operatorname{div} V \mathrm{~d} \mathscr{L}^{3}: V \in \Gamma(H), \operatorname{spt}(V) \subset \subset \Omega,\|V\| \leq 1\right\},
$$

where $\Gamma(H)$ contains all the smooth sections of the horizontal subbundle and $\operatorname{div} V$ is the divergence of vector fields on $\mathbf{R}^{3}$. One can show that, for every $V_{1}, V_{2} \in \mathscr{C}^{\infty}\left(\mathbf{R}^{3}\right)$,

$$
\operatorname{div}\left(V_{1} X+V_{2} Y\right)=X V_{1}+Y V_{2} .
$$

A set $E$ has locally finite perimeter if $P(E ; \Omega)<\infty$ for all $\Omega \subset \mathbf{H}$ open and bounded. If $E$ has locally finite perimeter, the function $\Omega \mapsto P(E ; \Omega)$ induces a Radon measure $|\partial E|$ on $\mathbf{H}^{1}$, which is concentrated on the so-called reduced boundary $\partial^{*} E \subset \partial E$. Moreover, up to a set of $|\partial E|$-measure zero and a rotation around the $z$-axis, $\partial^{*} E$ is the countable union of intrinsic graphs of $\mathscr{C}_{\mathbf{W}}^{1}$ functions. See [6] and [7] for further reading.

A measurable set $E$ has minimal perimeter if, for every bounded open set $\Omega \subset \mathbf{H}^{1}$ and every measurable set $F \subset \mathbf{H}^{1}$ with symmetric difference $E \Delta F \subset \subset \Omega$, we have

$$
P(E ; \Omega) \leq P(F ; \Omega) \text {. }
$$

In this case, the reduced boundary $\partial^{*} E$ of $E$ is called area-minimizing surface. We are interested in area minimizers that are global intrinsic graphs.

Conjecture 2.5. (Bernstein's problem) If $f \in \mathscr{C}_{\mathbf{W}}^{1}$ is such that $\Gamma_{f}$ is an areaminimizing surface, then $\Gamma_{f}$ is a vertical plane up to left-translations.

Such conjecture has been proven in the case $f \in \mathscr{C}^{1}\left(\mathbf{R}^{2}\right)$ in [9], while it has been presented a counterexample in [17] with $f \in \mathscr{C}^{0}\left(\mathbf{R}^{2}\right) \backslash \mathscr{C}_{\mathbf{W}}^{1}$.

For an open domain $\omega \subset \mathbf{R}^{2}$, set

$$
\Omega_{\omega}:=\{(0, \eta, \tau) *(\xi, 0,0):(\eta, \tau) \in \omega, \xi \in \mathbf{R}\} .
$$

If $f \in \mathscr{C}_{\mathbf{W}}^{1}$ and $E_{f}=\left\{(0, \eta, \tau) *(\xi, 0,0) \in \mathbf{R}^{2}, \xi \leq f(\eta, \tau)\right\}$, then

$$
P\left(E_{f} ; \Omega_{\omega}\right)=\int_{\omega} \sqrt{1+\left(\nabla^{f} f\right)^{2}} \mathrm{~d} \eta \mathrm{d} \tau .
$$

If $E_{f}$ has minimal perimeter, then, for every $g \in \mathscr{C}_{\mathbf{W}}^{1}$ with $\{f \neq g\} \subset \subset \omega$, it holds

$$
\int_{\omega} \sqrt{1+\left(\nabla^{f} f\right)^{2}} \mathrm{~d} \eta \mathrm{d} \tau \leq \int_{\omega} \sqrt{1+\left(\nabla^{g} g\right)^{2}} \mathrm{~d} \eta \mathrm{d} \tau
$$

It is not known whether the converse implication holds. 


\section{Lagrangian solutions to $\Delta^{f} f=0$}

For $f \in \mathscr{C}_{\mathbf{W}}^{1}$ and $v \in \mathscr{C}^{2}\left(\mathbf{R}^{2}\right)$, we define the differential operator

$$
\Delta^{f} v:=\partial_{\eta}^{2} v+2 f \partial_{\eta} \partial_{\tau} v+f^{2} \partial_{\tau}^{2} v+\nabla^{f} f \partial_{\tau} v
$$

Notice that, if $f \in \mathscr{C}^{2}\left(\mathbf{R}^{2}\right)$, then

$$
\Delta^{f} v=\nabla^{f}\left(\nabla^{f} v\right)
$$

The next lemma will be a fundamental tool for extending some results beyond the smooth case via approximation. The proof trivially follows from the explicit expressions of the differential operators $\nabla^{f}$ and $\Delta^{f}$.

Lemma 3.1. If $\left\{f_{k}\right\}_{k \in \mathbf{N}} \subset \mathscr{C}_{\mathbf{W}}^{1}$ and $\left\{v_{k}\right\}_{k \in \mathbf{N}} \subset \mathscr{C}^{2}\left(\mathbf{R}^{2}\right)$ are sequences converging to $f$ and $v$ in their respective spaces, then the sequences $\left\{\nabla^{f_{k}} v_{k}\right\}_{k \in \mathbf{N}}$ and $\left\{\Delta^{f_{k}} v_{k}\right\}_{k \in \mathbf{N}}$ converge to $\nabla^{f} v$ and $\Delta^{f} v$ uniformly on compact sets.

If $f \in \mathscr{C}^{2}\left(\mathbf{R}^{2}\right)$ is such that $\Gamma_{f}$ is a minimal surface in $\mathbf{H}$, then one shows that $f$ satisfies the differential equation (see [2])

$$
\nabla^{f}\left(\frac{\nabla^{f} f}{\sqrt{1+\left(\nabla^{f} f\right)^{2}}}\right)=0 .
$$

Equation (3) is equivalent, for $f \in \mathscr{C}^{2}\left(\mathbf{R}^{2}\right)$, to

$$
\Delta^{f} f=0 .
$$

For a generic $f \in \mathscr{C}_{\mathbf{W}}^{1}$, equation (4) has not the classical interpretation (2). However, using a "Lagrangian interpretation" of $\nabla^{f}\left(\nabla^{f} f\right)=0$, we give the following definition:

Definition 3.2. A function $f \in \mathscr{C}_{\mathbf{W}}^{1}$ satisfies $\Delta^{f} f=0$ in weak Lagrangian sense, if for every $p \in \mathbf{R}^{2}$ there is an integral curve $\gamma$ of $\nabla^{f}$ passing through $p$ such that $\nabla^{f} f$ is constant along $\gamma$.

If $f \in \mathscr{C}^{2}\left(\mathbf{R}^{2}\right)$ then $\Delta^{f} f=\nabla^{f}\left(\nabla^{f} f\right)=0$ if and only if $\nabla^{f} f$ is constant along all integral curves of $\nabla^{f}$, i.e., $\Delta^{f} f=0$ holds in a strong Lagrangian sense, see Remark 3.7.

Lemma 3.5 will characterize such functions by the integral curves of $\nabla^{f}$.

Lemma 3.3. Let $A, B \in \mathscr{C}^{0}(\mathbf{R})$. The map $\mathbf{R}^{2} \rightarrow \mathbf{R}^{2}$ given by

$$
G:(t, \zeta) \mapsto\left(t, \frac{A(\zeta)}{2} t^{2}+B(\zeta) t+\zeta\right)
$$

is a homeomorphism if and only if

(1) For all $\zeta, \zeta^{\prime} \in \mathbf{R}$

(1a) either $A(\zeta)=A\left(\zeta^{\prime}\right)$ and $B(\zeta)=B\left(\zeta^{\prime}\right)$,

(1b) or $2\left(A(\zeta)-A\left(\zeta^{\prime}\right)\right)\left(\zeta-\zeta^{\prime}\right)>\left(B(\zeta)-B\left(\zeta^{\prime}\right)\right)^{2}$.

(2) If there exists $\zeta_{0} \in \mathbf{R}$ such that $A\left(\zeta_{0}\right)>0$, then

$$
\limsup _{\zeta \rightarrow \infty}\left(\zeta-\frac{B(\zeta)^{2}}{2 A(\zeta)}\right)=+\infty \text {. }
$$

(3) If there exists $\zeta_{0} \in \mathbf{R}$ such that $A\left(\zeta_{0}\right)<0$, then

$$
\liminf _{\zeta \rightarrow-\infty}\left(\zeta-\frac{B(\zeta)^{2}}{2 A(\zeta)}\right)=-\infty
$$


Proof. Define $g(t, \zeta)=\frac{A(\zeta)}{2} t^{2}+B(\zeta) t+\zeta$, so that $G(t, \zeta)=(t, g(t, \zeta))$. We first show that $G$ is injective if and only if property (1) holds. For $\zeta^{\prime}>\zeta$, define the quadratic polynomial

$$
Q_{\zeta^{\prime}, \zeta}(t)=g\left(t, \zeta^{\prime}\right)-g(t, \zeta)=\frac{A\left(\zeta^{\prime}\right)-A(\zeta)}{2} t^{2}+\left(B\left(\zeta^{\prime}\right)-B(\zeta)\right) t+\left(\zeta^{\prime}-\zeta\right) .
$$

The map $G$ is injective if and only if for all $\zeta^{\prime}, \zeta \in \mathbf{R}$ with $\zeta^{\prime}>\zeta$ the polynomial $Q_{\zeta^{\prime}, \zeta}$ has no zeros. If $A\left(\zeta^{\prime}\right)=A(\zeta)$, then $Q_{\zeta^{\prime}, \zeta}$ is in fact linear, thus it has no zeros if and only if $B\left(\zeta^{\prime}\right)=B(\zeta)$ and we obtain property (1a). If $A\left(\zeta^{\prime}\right) \neq A(\zeta)$, then $Q_{\zeta^{\prime}, \zeta}$ has no zeros if and only if its discriminant is strictly negative, i.e., property (1b) holds.

Next, we assume that $G$ is injective, i.e., that property (1) holds, and we will show that $G$ is surjective if and only if properties (2) and (3) hold. By the Invariance of Domain Theorem, the fact that $G$ is surjective is equivalent to $G$ being a homeomorphism. Notice that, since $Q_{\zeta^{\prime}, \zeta}(0)=\zeta^{\prime}-\zeta>0$ for all $\zeta^{\prime}>\zeta$, we have

$$
\zeta^{\prime}>\zeta \quad \Longrightarrow \quad \forall t \in \mathbf{R} \quad g\left(t, \zeta^{\prime}\right)>g(t, \zeta) \text {. }
$$

Suppose that $G$ is surjective, hence a homeomorphism. Suppose $\zeta_{0} \in \mathbf{R}$ is such that $A\left(\zeta_{0}\right)>0$. By (1) we have that $A$ is monotone increasing, therefore $A(\zeta)>0$ for all $\zeta \geq \zeta_{0}$. It follows that if $\zeta \geq \zeta_{0}$ then

$$
\zeta-\frac{B(\zeta)^{2}}{2 A(\zeta)}=\inf _{t \in \mathbf{R}} g(t, \zeta)
$$

For $M \in \mathbf{R}$ define $K_{M}=\left\{(\eta, \tau) \in \mathbf{R}^{2}: g\left(\eta, \zeta_{0}\right) \leq \tau \leq M\right\}$. Since $A\left(\zeta_{0}\right)>0$, the set $K_{M}$ is compact (possibly empty) for all $M \in \mathbf{R}$. Next, for $\zeta \in \mathbf{R}$ define $U_{\zeta}=G(\mathbf{R} \times(-\infty, \zeta))=\{(\eta, \tau): \tau<g(\eta, \zeta)\}$. Since $G$ is surjective, the open sets $U_{\zeta}$ cover $\mathbf{R}^{2}$. Hence, there is $\zeta_{1} \geq \zeta_{0}$ such that $K_{M} \subset U_{\zeta_{1}}$. Using (5), we obtain

$$
\forall \zeta \geq \zeta_{1} \quad \inf _{t \in \mathbf{R}} g(t, \zeta) \geq M
$$

Since $M$ is arbitrary, we have proven (2). Property (3) is proven with a similar argument.

Now we prove the converse implication. Suppose that $A$ and $B$ satisfy properties (2) and (3). In order to prove that $G$ is surjective, we need only to prove that $\lim _{\zeta \rightarrow \infty} g(t, \zeta)=+\infty$ and $\lim _{\zeta \rightarrow-\infty} g(t, \zeta)=-\infty$, for every $t \in \mathbf{R}$.

If $A(\zeta)=A(0)$ for all $\zeta \geq 0$, then $g(t, \zeta)=g(t, 0)+\zeta$ and thus $\lim _{\zeta \rightarrow \infty} g(t, \zeta)=$ $+\infty$. If $A(\zeta) \leq 0$ for all $\zeta \in \mathbf{R}$, then there is $C>0$ such that $0 \leq A(\zeta)-A(0) \leq C$ for all $\zeta>0$. We may suppose $A(\zeta)>A(0)$ for $\zeta$ large enough. Thus, using $(1 b)$,

$$
\begin{aligned}
g(t, \zeta) & \geq \frac{A(0)}{2} t^{2}+B(0) t+\zeta+(B(\zeta)-B(0)) t \\
& \geq \frac{A(0)}{2} t^{2}+B(0) t+\zeta-|t| \sqrt{2(A(\zeta)-A(0)) \zeta} \\
& \geq \frac{A(0)}{2} t^{2}+B(0) t+\zeta-|t| \sqrt{2 C} \sqrt{\zeta}
\end{aligned}
$$

The limit $\lim _{\zeta \rightarrow \infty} g(t, \zeta)=+\infty$ follows. Finally, if $A\left(\zeta_{0}\right)>0$ for some $\zeta_{0} \in \mathbf{R}$, then for all $\zeta \geq \zeta_{0}$ we have $\inf _{t \in \mathbf{R}} g(t, \zeta)=\zeta-\frac{B(\zeta)^{2}}{2 A(\zeta)}$. Property (2) implies that $\lim _{\zeta \rightarrow \infty} g(t, \zeta)=+\infty$.

The limit $\lim _{\zeta \rightarrow-\infty} g(t, \zeta)=-\infty$ is deduced similarly from (3). 
Remark 3.4. If $A, B \in \mathscr{C}(\mathbf{R})$ satisfy properties (1), (2) and (3) of the previous Lemma 3.3, then the function $f$ defined by $f(G(t, \zeta))=\partial_{t} g(t, \zeta)=A(\zeta) t+B(\zeta)$ belongs to $\mathscr{C}_{\mathbf{W}}^{1}$ by Lemma 2.1. Moreover, the curves $t \mapsto g(t, \zeta)$ are integral curves of $\nabla^{f}$ along which $\nabla^{f} f(G(t, \zeta))=\partial_{t}^{2} g(t, \zeta)=A(\zeta)$ is constant. So, $\Delta^{f} f=0$ in weak Lagrangian sense. The graphs of these functions are examples of "graphical strips" as introduced in [4]. For example, for any $A \in \mathscr{C}^{0}(\mathbf{R})$ non-decreasing, we can define $g(t, \zeta):=A(\zeta) t^{2}+\zeta$ and we obtain a well defined $f \in \mathscr{C}_{\mathbf{W}}^{1}$ with $\Delta^{f} f=0$ given by

$$
f\left(t, A(\zeta) t^{2}+\zeta\right)=2 A(\zeta) t
$$

The converse also holds, as the next lemma shows.

Lemma 3.5. Let $f \in \mathscr{C}_{\mathbf{W}}^{1}$ satisfying $\Delta^{f} f=0$ in weak Lagrangian sense. Then the curves $t \mapsto(t, g(t, \zeta))$, where $\zeta \in \mathbf{R}$ and

$$
g(t, \zeta)=\frac{\nabla^{f} f(0, \zeta)}{2} t^{2}+f(0, \zeta) t+\zeta
$$

are the integral curves of $\nabla^{f}$ along which $\nabla^{f} f$ is constant. Moreover, the functions $\zeta \mapsto \nabla^{f} f(0, \zeta)$ and $\zeta \mapsto f(0, \zeta)$ satisfy the conditions (1), (2) and (3) in Lemma 3.3. In particular, $\tau \mapsto \nabla^{f} f(\eta, \tau)$ is non-decreasing, for all $\eta \in \mathbf{R}$.

Proof of Lemma 3.5. Given a function $g_{p}: I \rightarrow \mathbf{R}$ like in Lemma 2.1 along which $\nabla^{f} f$ is constant, we have $g_{p}^{\prime \prime}(t)=\nabla^{f} f(p)$ for all $t \in I$, i.e., $g_{p}$ is a polynomial of second degree. Moreover, such a $g_{p}$ is unique for every $p$, because it is completely determined by $f(p)$ and $\nabla^{f} f(p)$.

It follows that $g_{p}$ is defined on $\mathbf{R}$. Indeed, suppose $I=(a, b)$ and set $q=$ $\lim _{t \rightarrow b} p+\left(t, g_{p}(t)\right)$, which exists because $g_{p}$ is a polynomial. If $g_{q}: J \rightarrow \mathbf{R}$ is a function like in Lemma 2.1 along which $\nabla^{f} f$ is constant, then $g_{q}$ is uniquely determined by $f(q)$ and $\nabla^{f} f(q)$, where

$$
\begin{aligned}
f(q) & =\lim _{t \rightarrow b} f\left(p+\left(t, g_{p}(t)\right)\right)=\lim _{t \rightarrow b} g_{p}^{\prime}(t), \\
\nabla^{f} f(q) & =\lim _{t \rightarrow b} \nabla^{f} f\left(p+\left(t, g_{p}(t)\right)\right)=\lim _{t \rightarrow b} g_{p}^{\prime \prime}(t) .
\end{aligned}
$$

Hence, $g_{q}(t)=g_{p}(b+t)$ for $t<0$ and so $g_{p}$ can be extended beyond $b$. Similarly, we can extend $g_{p}$ to values below $a$.

If we consider $p=(0, \zeta)$, then $g_{p}(t)=g(t, \zeta)$, where $g(t, \zeta)$ is given in (6). If $p \in \mathbf{R}^{2}$, then the curve $t \mapsto p+\left(t, g_{p}(t)\right)$ intersects the axis $\{0\} \times \mathbf{R}$ at some point, and thus $g_{p}$ is of the form described in (6) up to a change of variables in $t$. We conclude that the map $(t, \zeta) \mapsto(t, g(t, \zeta))$ is a homeomorphism. Therefore, the conditions stated in Lemma 3.3 hold true.

Finally, since $\left(f(0, \zeta)-f\left(0, \zeta^{\prime}\right)\right)^{2} \geq 0$, then $\zeta \mapsto \nabla^{f} f(0, \zeta)$ is non-decreasing. Since $\nabla^{f} f(t, g(t, \zeta))=\nabla^{f} f(0, \zeta)$ and since, for $t \in \mathbf{R}$ fixed, the map $\zeta \mapsto g(t, \zeta)$ is a ordering-preserving homeomorphism $\mathbf{R} \rightarrow \mathbf{R}$, then the map $\tau \mapsto \nabla^{f} f(\eta, \tau)$ is non-decreasing as well, for all $\eta \in \mathbf{R}$.

Remark 3.6. Lemma 3.5 states in particular that, if $\Delta^{f} f=0$ in weak Lagrangian sense then $\Gamma_{f}$ is foliated by horizontal straight lines. Indeed, notice that any parabola $t \mapsto g(t, \zeta)$ in $\mathbf{R}^{2}$ lifts to a straight line in $\Gamma_{f}$. In [9, Theorem 3.5] Galli and Ritoré are able to prove that, if $f \in \mathscr{C}^{1}\left(\mathbf{R}^{2}\right)$ and if $\Gamma_{f}$ is a minimal surface in $\mathbf{H}$, then $\Gamma_{f}$ is foliated by horizontal straight lines, i.e., $\Delta^{f} f=0$ holds in weak Lagrangian sense. 
Remark 3.7. One may wonder wether Definition 3.2 for weak Lagrangian solutions to $\Delta^{f} f=0$ is equivalent to a stronger condition, namely that $\nabla^{f} f$ is constant along all integral curves of $\nabla^{f}$. This is the case when $f \in C^{1}\left(\mathbf{R}^{2}\right)$, because integral curves are unique at each point. The following example shows that strong and weak conditions are not equivalent. Indeed, there are functions for which the curves $t \mapsto(t, g(t, \zeta))$ described in Lemma 3.5 do not exhaust all the integral curves of $\nabla^{f}$.

Let $h \in \mathscr{C}^{2}(\mathbf{R})$ and define $k: \mathbf{R}^{2} \rightarrow \mathbf{R}$ by requiring that for each $s \in \mathbf{R}$ the function $t \mapsto k(t, s)$ is the unique polynomial of second degree with $k(s, s)=h(s)$, $\partial_{t} k(s, s)=h^{\prime}(s)$ and $\partial_{t}^{2} k(s, s)=h^{\prime \prime}(s)$. Explicitly, we have

$$
k(t, s)=\frac{h^{\prime \prime}(s)}{2} t^{2}+\left(h^{\prime}(s)-h^{\prime \prime}(s) s\right) t+h(s)-h^{\prime}(s) s+\frac{h^{\prime \prime}(s)}{2} s^{2} .
$$

If the map $K(t, s)=(t, k(t, s))$ is a homeomorphism $\mathbf{R}^{2} \rightarrow \mathbf{R}^{2}$, then we may define a function $f \in \mathscr{C}_{\mathbf{W}}^{1}$ by $f(K(t, s))=\partial_{t} k(t, s)$, as we did in Remark 3.4. Then $t \mapsto K(t, s)$ are integral curves of $\nabla^{f}$ and $\nabla^{f} f(K(t, s))=\partial_{t}^{2} k(t, s)=h^{\prime \prime}(s)$. It follows that $\Delta^{f} f=0$ holds in weak Lagrangian sense. However, $s \mapsto K(s, s)=(s, h(s))$ is an integral curve of $\nabla^{f}$, because $f(K(s, s))=h^{\prime}(s)$. Since $\nabla^{f} f(K(s, s))=h^{\prime \prime}(s)$, there is no need for $\nabla^{f} f$ to be constant along this curve.

As an example, consider $h(s)=s^{3}$, for which we have $k(t, s)=3 s t^{2}-3 s^{2} t+s^{3}$. We show that the map $K$ is in this case a homeomorphism. Define $\zeta(s)=s^{3}$, $A(\zeta(s))=6 s=6 \zeta^{1 / 3}, B(\zeta(s))=-3 s^{2}=-3 \zeta^{2 / 3}$ and the functions $g(t, \zeta)$ and $G(t, \zeta)$ as in Lemma 3.3. Since $K(t, s)=G(t, \zeta(s))$ and since $\zeta(\cdot)$ is a homeomorphism $\mathbf{R} \rightarrow \mathbf{R}$, we need only to show that $G$ is a homeomorphism $\mathbf{R}^{2} \rightarrow \mathbf{R}^{2}$, i.e., that the functions $A$ and $B$ satisfy all conditions of Lemma 3.3:

(1) Let $\zeta=\zeta(s), \zeta^{\prime}=\zeta\left(s^{\prime}\right) \in \mathbf{R}$. If $A(\zeta)=A\left(\zeta^{\prime}\right)$, then $s=s^{\prime}$ and thus $B(\zeta)=$ $B\left(\zeta^{\prime}\right)$. If instead $A(\zeta) \neq A\left(\zeta^{\prime}\right)$, then $s \neq s^{\prime}$ and thus

$$
\begin{aligned}
& 2\left(A(\zeta)-A\left(\zeta^{\prime}\right)\right)\left(\zeta-\zeta^{\prime}\right)-\left(B(\zeta)-B\left(\zeta^{\prime}\right)\right)^{2} \\
& =2\left(6 s-6 s^{\prime}\right)\left(s^{3}-s^{\prime 3}\right)-9\left(s^{\prime 2}-s^{2}\right)^{2}=3\left(s-s^{\prime}\right)^{4}>0 .
\end{aligned}
$$

$(2) \&(3)$ Since $\zeta-\frac{B(\zeta)^{2}}{2 A(\zeta)}=\frac{1}{4} s^{3}$ and since $\zeta \rightarrow \pm \infty$ if and only if $s \rightarrow \pm \infty$, then

$$
\lim _{\zeta \rightarrow+\infty} \zeta-\frac{B(\zeta)^{2}}{2 A(\zeta)}=\lim _{s \rightarrow+\infty} \frac{1}{4} s^{3}=+\infty
$$

and

$$
\lim _{\zeta \rightarrow-\infty} \zeta-\frac{B(\zeta)^{2}}{2 A(\zeta)}=\lim _{s \rightarrow-\infty} \frac{1}{4} s^{3}=-\infty
$$

The function $f$ can be explicitly computed as $f(\eta, \tau)=3 \eta^{2}-3\left(\tau-\eta^{3}\right)^{2 / 3}$. Finally, as we noticed before, $s \mapsto\left(s, s^{3}\right)$ is an integral curve of $\nabla^{f}$ and $\nabla^{f} f\left(s, s^{3}\right)=6 s$ is not constant.

Lemma 3.8. Let $f \in \mathscr{C}_{\mathbf{W}}^{1}$. If $\Delta^{f} f=0$ in weak Lagrangian sense, then there is a sequence $\left\{f_{k}\right\}_{k \in \mathbf{N}} \subset \mathscr{C}^{\infty}\left(\mathbf{R}^{2}\right)$ converging to $f$ in $\mathscr{C}_{\mathbf{W}}^{1}$ such that $\Delta^{f_{k}} f_{k}=0$ for all $k \in \mathbf{N}$.

Proof. Let $\left\{\rho_{\epsilon}\right\}_{\epsilon>0} \subset \mathscr{C}^{\infty}(\mathbf{R})$ be a family of mollifiers with $\operatorname{spt}\left(\rho_{\epsilon}\right) \subset[-\epsilon, \epsilon], \rho_{\epsilon} \geq$ $0, \rho_{\epsilon}(0)>0$ and $\int_{\mathbf{R}} \rho_{\epsilon}(r) \mathrm{d} r=1$. Fix $f \in \mathscr{C}_{\mathbf{W}}^{1}$ with $\Delta^{f} f=0$. Set $A(\zeta):=\nabla^{f} f(0, \zeta)$ and $B(\zeta):=f(0, \zeta)$. Define

$$
A_{\epsilon}(\zeta):=\int_{\mathbf{R}} \nabla^{f} f(0, \zeta-r) \rho_{\epsilon}(r) \mathrm{d} r
$$




$$
\begin{aligned}
B_{\epsilon}(\zeta) & :=\int_{\mathbf{R}} f(0, \zeta-r) \rho_{\epsilon}(r) \mathrm{d} r, \\
g_{\epsilon}(t, \zeta) & :=\frac{A_{\epsilon}(\zeta)}{2} t^{2}+B_{\epsilon}(\zeta) t+\zeta .
\end{aligned}
$$

We claim that, for all $\epsilon>0$, all conditions stated in Lemma 3.3 hold for $A_{\epsilon}$ and $B_{\epsilon}$. Let $\zeta, \zeta^{\prime} \in \mathbf{R}$ with $\zeta<\zeta^{\prime}$. First, suppose that $A_{\epsilon}(\zeta)=A_{\epsilon}\left(\zeta^{\prime}\right)$. Notice that $A(\zeta-r)-A\left(\zeta^{\prime}-r\right) \leq 0$ for all $r \in \mathbf{R}$, because $A$ is non-decreasing. Thus, we deduce from

$$
0=A_{\epsilon}(\zeta)-A_{\epsilon}\left(\zeta^{\prime}\right)=\int_{\mathbf{R}}\left(A(\zeta-r)-A\left(\zeta^{\prime}-r\right)\right) \rho_{\epsilon}(r) \mathrm{d} r
$$

that $\left(B(\zeta-r)-B\left(\zeta^{\prime}-r\right)\right) \rho_{\epsilon}(r)=0$ for all $r \in \mathbf{R}$ and therefore that $B_{\epsilon}(\zeta)=B_{\epsilon}\left(\zeta^{\prime}\right)$, i.e., (1a) holds.

Second, suppose that $A_{\epsilon}(\zeta) \neq A_{\epsilon}\left(\zeta^{\prime}\right)$. Using Jensen's inequality, we have

$$
\begin{aligned}
& 2\left(A_{\epsilon}(\zeta)-A_{\epsilon}\left(\zeta^{\prime}\right)\right)\left(\zeta-\zeta^{\prime}\right) \\
& =\int_{\mathbf{R}} 2\left(A(\zeta-r)-A\left(\zeta^{\prime}-r\right)\right)\left((\zeta-r)-\left(\zeta^{\prime}-r\right)\right) \rho_{\epsilon}(r) \mathrm{d} r \\
& >\int_{\mathbf{R}}\left(B(\zeta-r)-B\left(\zeta^{\prime}-r\right)\right)^{2} \rho_{\epsilon}(r) \mathrm{d} r \geq\left(\int_{\mathbf{R}}\left(B(\zeta-r)-B\left(\zeta^{\prime}-r\right)\right) \rho_{\epsilon}(r) \mathrm{d} r\right)^{2} .
\end{aligned}
$$

So, condition (1b) is also verified.

Suppose that $A_{\epsilon}\left(\zeta_{0}\right)>0$ for some $\zeta_{0} \in \mathbf{R}$. By the monotonicity of $A$ and the positivity of $\rho_{\epsilon}$, we may assume $A\left(\zeta_{0}\right)>0$. Let $M>0$. Since property (2) of Lemma 3.3 holds for $A$, there is $\zeta_{1}>\zeta_{0}$ so that for all $\zeta>\zeta_{1}$

$$
M<\zeta-\frac{B(\zeta)^{2}}{2 A(\zeta)}=\frac{2 A(\zeta) \zeta-B(\zeta)^{2}}{2 A(\zeta)} .
$$

Using Jensen inequality, we have for all $\zeta>\zeta_{1}+\epsilon$

$$
\begin{aligned}
& 2 A_{\epsilon}(\zeta) \zeta-B_{\epsilon}(\zeta)^{2} \geq \int_{\mathbf{R}}\left(2 \zeta A(\zeta-r)-B(\zeta-r)^{2}\right) \rho_{\epsilon}(r) \mathrm{d} r \\
& =2 \int_{\mathbf{R}} A(\zeta-r) r \rho_{\epsilon}(r) \mathrm{d} r+\int_{\mathbf{R}}\left(2(\zeta-r) A(\zeta-r)-B(\zeta-r)^{2}\right) \rho_{\epsilon}(r) \mathrm{d} r \\
& \geq-2 \epsilon \int_{\mathbf{R}} A(\zeta-r) \rho_{\epsilon}(r) \mathrm{d} r+2 M \int_{\mathbf{R}} A(\zeta-r) \rho_{\epsilon}(r) \mathrm{d} r=2 A_{\epsilon}(\zeta)(M-\epsilon) .
\end{aligned}
$$

Thus, $M-\epsilon<\zeta-\frac{B_{\epsilon}(\zeta)^{2}}{2 A_{\epsilon}(\zeta)}$ for all $\zeta>\zeta_{1}+\epsilon$. Since $M$ was arbitrary, we obtain property (2) of Lemma 3.3. Property (3) can be similarly obtained.

The functions $G_{\epsilon}: \mathbf{R}^{2} \rightarrow \mathbf{R}^{2}, G_{\epsilon}(t, \zeta):=\left(t, g_{\epsilon}(t, \zeta)\right)$, are homeomorphisms and, as $\epsilon \rightarrow 0$, they converge to $G_{0}$ uniformly on compact sets. It follows that $G_{\epsilon}^{-1}$ also converge to $G_{0}^{-1}$, as $\epsilon \rightarrow 0$.

For $\epsilon>0$, define $f_{\epsilon} \in \mathscr{C}^{\infty}\left(\mathbf{R}^{2}\right)$ via

$$
f_{\epsilon}\left(t, g_{\epsilon}(t, \zeta)\right)=A_{\epsilon}(\zeta) t+B_{\epsilon}(\zeta) .
$$

By the continuity of $G_{\epsilon}$ and $G_{\epsilon}^{-1}$ in $\epsilon, f_{\epsilon}$ and $\nabla^{f_{\epsilon}} f_{\epsilon}$ converge to $f_{0}$ and $\nabla^{f_{0}} f_{0}$ uniformly on compact sets. Finally, $\Delta^{f_{\epsilon}} f_{\epsilon}=0$ by construction. 


\section{A Lagrangian approach to contact variations}

Proposition 4.1. Let $\phi=\left(\phi_{1}, \phi_{2}\right): \mathbf{R}^{2} \rightarrow \mathbf{R}^{2}$ be a $\mathscr{C}^{\infty}$-diffeomorphism. Let $f \in \mathscr{C}_{\mathbf{W}}^{1}$ and assume

$$
\nabla^{f} \phi_{1}(p) \neq 0 \quad \forall p \in \mathbf{R}^{2}
$$

Define $\bar{f}: \mathbf{R}^{2} \rightarrow \mathbf{R}$ as

$$
\bar{f} \circ \phi=\frac{\nabla^{f} \phi_{2}}{\nabla^{f} \phi_{1}}
$$

Then $\bar{f} \in \mathscr{C}_{\mathbf{W}}^{1}$ and

$$
\nabla^{\bar{f}} \bar{f} \circ \phi=\frac{\Delta^{f} \phi_{2}}{\left(\nabla^{f} \phi_{1}\right)^{2}}-\frac{\nabla^{f} \phi_{2}}{\left(\nabla^{f} \phi_{1}\right)^{3}} \Delta^{f} \phi_{1} .
$$

Notice that, if $f \in \mathscr{C}^{1}\left(\mathbf{R}^{2}\right)$, then $\bar{f} \in \mathscr{C}^{1}\left(\mathbf{R}^{2}\right)$ as well.

Remark 4.2. If $\left\{\phi^{\epsilon}\right\}_{\epsilon>0}$ is a smooth one-parameter family of diffeomorphisms $\phi^{\epsilon}: \mathbf{R}^{2} \rightarrow \mathbf{R}^{2}$ with $\phi^{0}=\mathrm{Id}$, then, for $\epsilon>0$ small enough, the functions $f_{\epsilon}$ defined by

$$
f_{\epsilon} \circ \phi^{\epsilon}=\frac{\nabla^{f} \phi_{2}^{\epsilon}}{\nabla^{f} \phi_{1}^{\epsilon}}
$$

belong to $\mathscr{C}_{\mathbf{W}}^{1}$ and converge to $f$ in $\mathscr{C}_{\mathbf{W}}^{1}$.

Proof. The idea is to transform via $\phi$ the integral curves of $\nabla^{f}$ into the ones of $\nabla^{\bar{f}}$. Fix $p=(\eta, \tau)$, let $q:=(\bar{\eta}, \bar{\tau}):=\phi(p)$ and let $g_{p}: I \rightarrow \mathbf{R}$ be like in Lemma 2.1 . Thanks to the condition $\nabla^{f} \phi_{1} \neq 0$ and the Implicit Function Theorem, there exist two $\mathscr{C}^{2}$-function $s: I \rightarrow \mathbf{R}$ and $\bar{g}_{q}: s(I) \rightarrow \mathbf{R}$, such that

$$
q+\left(s, \bar{g}_{q}(s)\right)=\phi\left(p+\left(t, g_{p}(t)\right), \quad \forall t \in I .\right.
$$

Therefore

$$
\left\{\begin{array}{l}
s(t)=\phi_{1}\left(\eta+t, \tau+g_{p}(t)\right)-\bar{\eta}, \\
\bar{g}_{q}(s(t))=\phi_{2}\left(\eta+t, \tau+g_{p}(t)\right)-\bar{\tau} .
\end{array}\right.
$$

We define

$$
\bar{f}(q):=\bar{g}_{q}^{\prime}(0)
$$

Notice that this value does not depend on the choice of $g_{p}$, as far as $t \mapsto\left(t, g_{p}(t)\right)$ is an integral curve of $\nabla^{f}$.

We want to write $\bar{g}_{q}^{\prime}(0)$. Set

$$
p_{t}:=\left(\eta+t, \tau+g_{p}(t)\right) .
$$

First

$$
\begin{aligned}
\frac{\mathrm{d}}{\mathrm{d} t} s(t) & =\partial_{\eta} \phi_{1}\left(p_{t}\right)+\partial_{\tau} \phi_{1}\left(p_{t}\right) g_{p}^{\prime}(t)=\nabla^{f} \phi_{1}\left(p_{t}\right), \\
\frac{\mathrm{d}}{\mathrm{d} t} \bar{g}_{q}(s(t)) & =\partial_{\eta} \phi_{2}\left(p_{t}\right)+\partial_{\tau} \phi_{2}\left(p_{t}\right) g_{p}^{\prime}(t)=\nabla^{f} \phi_{2}\left(p_{t}\right) .
\end{aligned}
$$

Since

we have for $s=0=t$

$$
\frac{\mathrm{d}}{\mathrm{d} t} \bar{g}_{q}(s(t))=\bar{g}_{q}^{\prime}(s(t)) \cdot \frac{\mathrm{d}}{\mathrm{d} t} s(t),
$$

$$
\bar{f}(q)=\frac{\nabla^{f} \phi_{2}(p)}{\nabla^{f} \phi_{1}(p)} .
$$


$\nabla^{\bar{f}} \bar{f}(q)$ is the derivative of $\bar{f}$ along the curve $q+\left(s, \bar{g}_{q}(s)\right)$ at $s=0$, i.e.,

$$
\nabla^{\bar{f}} \bar{f}(q)=\bar{g}_{q}^{\prime \prime}(0) \text {. }
$$

As above, we want to write down $\bar{g}_{q}^{\prime \prime}(0)$ in a more explicit way.

$$
\begin{aligned}
\left.\frac{\mathrm{d}^{2}}{\mathrm{~d} t^{2}} s(t)\right|_{t=0}= & \partial_{\eta}^{2} \phi_{1}(p)+\partial_{\tau} \partial_{\eta} \phi_{1}(p) f(p) \\
& +\partial_{\eta} \partial_{\tau} \phi_{1}(p) f(p)+\partial_{\tau}^{2} \phi_{1}(p)(f(p))^{2}+\partial_{\tau} \phi_{1}(p) \nabla^{f} f(p)=\Delta^{f} \phi_{1}(p), \\
\left.\frac{\mathrm{d}^{2}}{\mathrm{~d} t^{2}} \bar{g}_{q}(s(t))\right|_{t=0}= & \partial_{\eta}^{2} \phi_{2}(p)+\partial_{\tau} \partial_{\eta} \phi_{2}(p) f(p) \\
& +\partial_{\eta} \partial_{\tau} \phi_{2}(p) f(p)+\partial_{\tau}^{2} \phi_{2}(p)(f(p))^{2}+\partial_{\tau} \phi_{2}(p) \nabla^{f} f(p)=\Delta^{f} \phi_{2}(p)
\end{aligned}
$$

Since

$$
\frac{\mathrm{d}^{2}}{\mathrm{~d} t^{2}} \bar{g}_{q}(s(t))=\bar{g}_{q}^{\prime \prime}(s(t)) \cdot\left(\frac{\mathrm{d}}{\mathrm{d} t} s(t)\right)^{2}+\bar{g}_{q}^{\prime}(s(t)) \cdot \frac{\mathrm{d}^{2}}{\mathrm{~d} t^{2}} s(t)
$$

we have

$$
\begin{aligned}
\nabla^{\bar{f}} \bar{f}(q) & =\bar{g}_{q}^{\prime \prime}(0)=\frac{\left.\frac{\mathrm{d}^{2}}{\mathrm{~d} t^{2}} \bar{g}_{q}\right|_{t=0}-\left.\bar{g}_{q}^{\prime}(0) \cdot \frac{\mathrm{d}^{2}}{\mathrm{~d} t^{2}} s\right|_{t=0}}{\left(\left.\frac{\mathrm{d}}{\mathrm{d} t} s\right|_{t=0}\right)^{2}} \\
& =\frac{1}{\left(\nabla^{f} \phi_{1}(p)\right)^{2}} \cdot\left(\Delta^{f} \phi_{2}(p)-\frac{\nabla^{f} \phi_{2}(p)}{\nabla^{f} \phi_{1}(p)} \cdot \Delta^{f} \phi_{1}(p)\right) .
\end{aligned}
$$

By Lemma 2.1, the function $\bar{f}$ belongs to $\mathscr{C}_{\mathbf{W}}^{1}$.

\section{Contact transformations}

A diffeomorphism $\Phi: \mathbf{H} \rightarrow \mathbf{H}$ is a contact diffeomorphism if $\mathrm{d} \Phi(H) \subset H$, see [3, 12]. Contact diffeomorphisms are the only diffeomorphisms that preserve the subRiemannian perimeter.

Proposition 5.1. Let $\Phi: \mathbf{H} \rightarrow \mathbf{H}$ be a diffeomorphism of class $\mathscr{C}^{2}$. If, for all $E \subset \mathbf{H}$ measurable and all $\Omega \subset \mathbf{H}$ open, it holds

$$
P(E ; \Omega)<\infty \quad \Longrightarrow \quad P(\Phi(E) ; \Phi(\Omega))<\infty,
$$

then $\Phi$ is contact.

We will show in this section that any variation of an intrinsic graph $\Gamma_{f}$ via contact diffeomorphisms is equivalent to a variation of $f$ via the transformations of Proposition 4.1 and Remark 4.2.

Proposition 5.2. Let $\phi: \mathbf{R}^{2} \rightarrow \mathbf{R}^{2}$ be a $\mathscr{C}^{\infty}$-diffeomorphism and $f, \bar{f} \in \mathscr{C}_{\mathbf{W}}^{1}$ as in Proposition 4.1. Then there is a contact diffeomorphism $\Phi: \Omega \rightarrow \Phi(\Omega)$, where $\Omega$ and $\Phi(\Omega)$ are open subsets of $\mathbf{H}$ with $\Gamma_{f} \subset \Omega$, such that $\Phi\left(\Gamma_{f}\right)=\Gamma_{\bar{f}}$.

Proposition 5.3. Let $\Phi^{\epsilon}: \mathbf{H} \rightarrow \mathbf{H}, \epsilon \in \mathbf{R}$, be a smooth one-parameter family of contact diffeomorphisms such that there is a compact set $K \subset \mathbf{H}$ with $\left.\Phi^{\epsilon}\right|_{\mathbf{H} \backslash K}=\mathrm{Id}$ for all $\epsilon$ and $\Phi^{0}=$ Id. Let $f \in \mathscr{C}^{\infty}\left(\mathbf{R}^{2}\right)$. Then there is $\epsilon_{0}>0$ such that for all $\epsilon$ with $|\epsilon|<\epsilon_{0}$, the maps $\phi^{\epsilon}: \mathbf{R}^{2} \rightarrow \mathbf{R}^{2}$,

$$
\phi^{\epsilon}(p):=\pi_{X} \circ \Phi^{\epsilon}(p * f(p)),
$$

form a smooth family of $\mathscr{C}^{\infty}$-diffeomorphism of $\mathbf{R}^{2}$. Moreover, if $f^{\epsilon}$ is the function defined via $f$ and $\phi^{\epsilon}$ as in Proposition 4.1, then

$$
\Phi^{\epsilon}\left(\Gamma_{f}\right)=\Gamma_{f^{\epsilon}} .
$$


5.1. Proof of Proposition 5.1. We use an argument by contradiction. Assume that $\Phi$ is not a contact diffeomorphism. Then there is an open and bounded set $\Omega \subset \mathbf{H}$ such that for all $p \in \Omega$ it holds $\mathrm{d} \Phi\left(H_{p}\right) \not \subset H_{\Phi(p)}$. Thanks to the following lemma and Remark 2.3, we get a contradiction with the property (9).

Lemma 5.4. Let $\Phi: \mathbf{H} \rightarrow \mathbf{H}$ be a diffeomorphism of class $\mathscr{C}^{2}$. Let $\Omega \subset \mathbf{H}$ be an open and bounded set such that for all $p \in \Omega$

$$
\mathrm{d} \Phi\left(H_{p}\right) \not \subset H_{\Phi(p)} \text {. }
$$

Let $E \subset \mathbf{H}$ be measurable. If $P(E ; \Omega)<\infty$ and $P(\Phi(E) ; \Phi(\Omega))<\infty$, then $E$ has finite Riemannian perimeter in $\Omega$.

Proof. We extend the scalar product $\langle\cdot, \cdot\rangle$ to the whole $T \mathbf{H}$ in such a way that $(X, Y, Z)$ is an orthonormal frame. The Riemannian perimeter is defined as

$$
P_{\mathscr{R}}(E ; \Omega):=\sup \left\{\int_{E} \operatorname{div} U \mathrm{~d} \mathscr{L}^{3}: U \in \operatorname{Vec}(T \mathbf{H}), \operatorname{spt} U \subset \subset \Omega,\|U\| \leq 1\right\} .
$$

Let $U \in \operatorname{Vec}(T \mathbf{H})$ with $\operatorname{spt}(U) \subset \subset \Omega$ and $\|U\| \leq 1$. Then there are $V, W \in$ $\operatorname{Vec}(T \mathbf{H})$ with $\operatorname{spt}(V) \cup \operatorname{spt}(W)=\operatorname{spt}(U), V+W=U, V(p) \in H_{p}$ for all $p,\|V\| \leq K$ and $\|W\| \leq K$, and $\Phi_{*} W(p) \in H_{p}$ for all $p$, where $K \geq 0$ depends on $\Phi$ and $\Omega$, but not on $U$.

Remind that, if $W$ is a smooth vector field on $\mathbf{H}$, then ${ }^{2}$

$$
\operatorname{div}\left(\Phi_{*} W\right)=\operatorname{div}(W) \circ \Phi^{-1} \cdot J\left(\Phi^{-1}\right) .
$$

Therefore

$$
\int_{E} \operatorname{div} W \mathrm{~d} \mathscr{L}^{3}=\int_{\Phi(E)}(\operatorname{div} W) \circ \Phi^{-1} J \Phi^{-1} \mathrm{~d} \mathscr{L}^{3}=\int_{\Phi(E)} \operatorname{div}\left(\Phi_{*} W\right) \mathrm{d} \mathscr{L}^{3} .
$$

Moreover, since $\Omega$ is bounded, we can assume $\|\mathrm{d} \Phi(v)\| \leq K\|v\|$ for all $v \in T \Omega$, where $K \geq 0$ is the same constant as above. Therefore

$$
\begin{aligned}
\int_{E} \operatorname{div} U \mathrm{~d} \mathscr{L}^{3} & =\int_{E} \operatorname{div} V \mathrm{~d} \mathscr{L}^{3}+\int_{E} \operatorname{div} W \mathrm{~d} \mathscr{L}^{3} \\
& =\int_{E} \operatorname{div} V \mathrm{~d} \mathscr{L}^{3}+\int_{\Phi(E)} \operatorname{div}\left(\Phi_{*} W\right) \mathrm{d} \mathscr{L}^{3} \\
& \leq K P(E ; \Omega)+K^{2} P(\Phi(E) ; \Phi(\Omega)) .
\end{aligned}
$$

This implies that $P_{\mathscr{R}}(E ; \Omega) \leq K P(E ; \Omega)+K^{2} P(\Phi(E) ; \Phi(\Omega))<\infty$.

5.2. Proof of Proposition 5.2. In this case our choice of coordinates is not helpful. So, we consider the exponential coordinates of second kind $(\xi, \eta, \tau) \mapsto$ $\exp (\eta B+\tau C) * \exp (\xi A)$, using the notation of Section 2.1.

We define the map $\Phi$ as

$$
\Phi(\xi, \eta, \tau):=\left(\frac{\nabla^{\xi} \phi_{2}}{\nabla^{\xi} \phi_{1}}(\eta, \tau), \phi_{1}(\eta, \tau), \phi_{2}(\eta, \tau)\right)
$$

Clearly, $\Phi$ is well defined and smooth on the open set

$$
\Omega:=\left\{(\xi, \eta, \tau): \nabla^{\xi} \phi_{1}(\eta, \tau) \neq 0\right\}
$$

\footnotetext{
${ }^{2}$ A sketch of the proof of this formula: it is clearer to show the dual formula $\operatorname{div}\left(\Phi^{*} W\right)=$ $\operatorname{div}(W) \circ \Phi \cdot J(\Phi)$; consider $W$ as a 2 -form and the divergence as the exterior derivative d; remind that $\mathrm{d} \Phi^{*}=\Phi^{*} \mathrm{~d}$; the formula follows.
} 
$\Gamma_{f} \subset \Omega$ by the hypothesis of Proposition 4.1 and $\Phi\left(\Gamma_{f}\right)=\Gamma_{\bar{f}}$. In these coordinates, the differential of $\Phi$ is

$$
\mathrm{d} \Phi(\xi, \eta, \tau)=\left(\begin{array}{ccc}
\partial_{\xi}\left(\frac{\nabla^{\xi} \phi_{2}}{\nabla^{\xi} \phi_{1}}\right) & \partial_{\eta}\left(\frac{\nabla^{\xi} \phi_{2}}{\nabla^{\xi} \phi_{1}}\right) & \partial_{\tau}\left(\frac{\nabla^{\xi} \phi_{2}}{\nabla^{\xi} \phi_{1}}\right) \\
0 & \partial_{\eta} \phi_{1} & \partial_{\tau} \phi_{1} \\
0 & \partial_{\eta} \phi_{2} & \partial_{\tau} \phi_{2}
\end{array}\right)
$$

Since $\phi$ is a diffeomorphism, $\Phi$ is a diffeomorphism if and only if $\partial_{\xi}\left(\frac{\nabla^{\xi} \phi_{2}}{\nabla^{\xi} \phi_{1}}\right) \neq 0$. A short computation shows that

$$
\partial_{\xi}\left(\frac{\nabla^{\xi} \phi_{2}}{\nabla^{\xi} \phi_{1}}\right)=\frac{\operatorname{det}(\mathrm{d} \phi)}{\left(\nabla^{\xi} \phi_{1}\right)^{2}}
$$

which is non-zero.

Now, we need to show that $\Phi$ is a contact diffeomorphism. In this system of coordinates, the left-invariant vector fields $X, Y, Z$ are written as

$$
\tilde{X}(\xi, \eta, \tau)=\partial_{\xi}, \quad \tilde{Y}(\xi, \eta, \tau)=\partial_{\eta}+\xi \partial_{\tau}, \quad \tilde{Z}(\xi, \eta, \tau)=\partial_{\tau} .
$$

We have

$$
\begin{aligned}
\mathrm{d} \Phi(\tilde{X}(\xi, \eta, \tau)) & =\partial_{\xi}\left(\frac{\nabla^{\xi} \phi_{2}}{\nabla^{\xi} \phi_{1}}\right) \tilde{X}(\Phi(\xi, \eta, \tau)), \\
\mathrm{d} \Phi(\tilde{Y}(\xi, \eta, \tau)) & =\nabla^{\xi}\left(\frac{\nabla^{\xi} \phi_{2}}{\nabla^{\xi} \phi_{1}}\right) \tilde{X}(\Phi(\xi, \eta, \tau))+\nabla^{\xi} \phi_{1} \tilde{Y}(\Phi(\xi, \eta, \tau)) .
\end{aligned}
$$

Therefore, $\mathrm{d} \Phi(H) \subset H$.

5.3. Proof of Proposition 5.3. The functions $\phi^{\epsilon}: \mathbf{R}^{2} \rightarrow \mathbf{R}^{2}$ are well defined and smooth for all $\epsilon \in \mathbf{R}$. Since $\Phi^{\epsilon}$ and all its derivative converge to Id uniformly on $\mathbf{H}$, there exists $\epsilon_{0}>0$ such that for all $\epsilon$ with $|\epsilon|<\epsilon_{0}$, the vector field $X$ is not tangent to $\Phi^{\epsilon}\left(\Gamma_{f}\right)$ at any point. Therefore, $\operatorname{det}\left(\mathrm{d} \phi^{\epsilon}\right) \neq 0$ for all such $\epsilon$. Since $\left.\phi^{\epsilon}\right|_{\pi_{X}(K)}=\mathrm{Id}, \phi^{\epsilon}$ is a covering map and therefore it is a smooth diffeomorphism.

The last statement is a direct consequence Lemma 2.2.

\section{First contact variation}

Similar formulas for the first and the second variation for the sub-Riemannian perimeter in the Heisenberg group can be found in $[4,5,8,15]$.

In all the formulas below, we set $\psi:=\nabla^{f} f$.

Proposition 6.1. Let $f \in \mathscr{C}_{\mathbf{W}}^{1}$ be such that $\Gamma_{f}$ is an area-minimizing surface. Then for all $V_{1}, V_{2} \in \mathscr{C}_{c}^{\infty}\left(\mathbf{R}^{2}\right)$ it holds

$$
0=\int_{\mathbf{R}^{2}}\left[\frac{\psi}{\sqrt{1+\psi^{2}}}\left(-2 \psi \cdot \nabla^{f} V_{1}-f \cdot \Delta^{f} V_{1}\right)+\sqrt{1+\psi^{2}} \partial_{\eta} V_{1}\right] \mathrm{d} \eta \mathrm{d} \tau
$$

and

$$
0=\int_{\mathbf{R}^{2}}\left[\frac{\psi}{\sqrt{1+\psi^{2}}} \Delta^{f} V_{2}+\sqrt{1+\psi^{2}} \partial_{\tau} V_{2}\right] \mathrm{d} \eta \mathrm{d} \tau
$$

Proposition 6.2. Let $f \in \mathscr{C}^{\infty}\left(\mathbf{R}^{2}\right)$ be such that for all $V_{2} \in \mathscr{C}_{c}^{\infty}\left(\mathbf{R}^{2}\right)$ the equation (11) holds. Then (10) holds as well for all $V_{1} \in \mathscr{C}_{c}^{\infty}\left(\mathbf{R}^{2}\right)$. 
Proposition 6.3. A function $f \in \mathscr{C}^{\infty}\left(\mathbf{R}^{2}\right)$ satisfies (11) for all $V_{2} \in \mathscr{C}_{c}^{\infty}\left(\mathbf{R}^{2}\right)$ if and only if

$$
\left(\nabla^{f}+2 \partial_{\tau} f\right) \nabla^{f}\left(\frac{\psi}{\sqrt{1+\psi^{2}}}\right)=0 .
$$

6.1. Proof of Proposition 6.1. Let $f \in \mathscr{C}_{\mathbf{W}}^{1}, \omega \subset \mathbf{R}^{2}$ an open and bounded set and $V=\left(V_{1}, V_{2}\right): \mathbf{R}^{2} \rightarrow \mathbf{R}^{2}$ a smooth vector field with spt $V \subset \subset \omega$. Let $\phi^{\epsilon}: \mathbf{R}^{2} \rightarrow$ $\mathbf{R}^{2}$ be a smooth one-parameter family of diffeomorphism such that $\left\{\phi^{\epsilon} \neq \operatorname{Id}\right\} \subset \operatorname{spt} V$ for all $\epsilon>0$ and, for all $p \in \mathbf{R}^{2}$,

$$
\left\{\begin{array}{l}
\phi^{0}(p)=p \\
\left.\partial_{\epsilon} \phi^{\epsilon}(p)\right|_{\epsilon=0}=V(p) .
\end{array}\right.
$$

Notice that $\nabla^{f} \phi_{1}^{\epsilon}=\partial_{\eta} \phi_{1}^{\epsilon}+f \partial_{\tau} \phi_{1}^{\epsilon}$ is not zero for $\epsilon$ small enough, because $\nabla^{f} \phi_{1}^{\epsilon}$ converges to 1 uniformly as $\epsilon \rightarrow 0$. Hence, by Proposition 4.1, there is an interval $I=(-\hat{\epsilon}, \hat{\epsilon})$ such that the function given by

$$
f_{\epsilon} \circ \phi^{\epsilon}=\frac{\nabla^{f} \phi_{2}^{\epsilon}}{\nabla^{f} \phi_{1}^{\epsilon}}
$$

is well defined for all $\epsilon \in I$. Define $\gamma: I \rightarrow \mathbf{R}$ as

$$
\gamma(\epsilon):=\int_{\omega} \sqrt{1+\left(\nabla^{f_{\epsilon}} f_{\epsilon}\right)^{2}} \mathrm{~d} \eta \mathrm{d} \tau=\int_{\omega} \sqrt{1+\left(\left(\nabla^{f_{\epsilon}} f_{\epsilon}\right) \circ \phi^{\epsilon}\right)^{2}} J_{\phi^{\epsilon}} \mathrm{d} \eta \mathrm{d} \tau
$$

where we performed a change of coordinates via $\phi^{\epsilon}$ and

$$
J_{\phi^{\epsilon}}=\partial_{\eta} \phi_{1}^{\epsilon} \partial_{\tau} \phi_{2}^{\epsilon}-\partial_{\tau} \phi_{1}^{\epsilon} \partial_{\eta} \phi_{2}^{\epsilon}
$$

is the Jacobian of $\phi^{\epsilon}$. Using equality (8) and Lemma 3.1, it is immediate to see that $\gamma$ is continuous.

Lemma 6.4. The function $\gamma: I \rightarrow \mathbf{R}$ is continuously differentiable and

$$
\begin{aligned}
\gamma^{\prime}(\epsilon)=\int_{\omega} & {\left[\frac{\left(\left(\nabla^{f_{\epsilon}} f_{\epsilon}\right) \circ \phi^{\epsilon}\right)}{\sqrt{1+\left(\left(\nabla^{f_{\epsilon}} f_{\epsilon}\right) \circ \phi^{\epsilon}\right)^{2}}} A_{f}(\epsilon) J_{\phi^{\epsilon}}\right.} \\
& \left.+\sqrt{1+\left(\left(\nabla^{f_{\epsilon}} f_{\epsilon}\right) \circ \phi^{\epsilon}\right)^{2}} \partial_{\epsilon} J_{\phi^{\epsilon}}\right] \mathrm{d} \eta \mathrm{d} \tau
\end{aligned}
$$

where

$$
\begin{aligned}
A_{f}(\epsilon):= & \frac{\Delta^{f} \partial_{\epsilon} \phi_{2}^{\epsilon}}{\left(\nabla^{f} \phi_{1}^{\epsilon}\right)^{2}}-2 \frac{\Delta^{f} \phi_{2}^{\epsilon}}{\left(\nabla^{f} \phi_{1}^{\epsilon}\right)^{3}} \nabla^{f} \partial_{\epsilon} \phi_{1}^{\epsilon} \\
& -\frac{\nabla^{f} \partial_{\epsilon} \phi_{2}^{\epsilon}}{\left(\nabla^{f} \phi_{1}^{\epsilon}\right)^{3}} \Delta^{f} \phi_{1}^{\epsilon}+3 \frac{\nabla^{f} \phi_{2}^{\epsilon}}{\left(\nabla^{f} \phi_{1}^{\epsilon}\right)^{4}} \nabla^{f} \partial_{\epsilon} \phi_{1}^{\epsilon} \cdot \Delta^{f} \phi_{1}^{\epsilon}-\frac{\nabla^{f} \phi_{2}^{\epsilon}}{\left(\nabla^{f} \phi_{1}^{\epsilon}\right)^{3}} \Delta^{f} \partial_{\epsilon} \phi_{1}^{\epsilon} .
\end{aligned}
$$

Proof of Lemma 6.4. First, suppose $f \in \mathscr{C}^{\infty}\left(\mathbf{R}^{2}\right)$. Then $\gamma \in \mathscr{C}^{\infty}(I)$ and

$$
\begin{aligned}
\gamma^{\prime}(\epsilon)=\int_{\omega} & {\left[\frac{\left(\left(\nabla^{f_{\epsilon}} f_{\epsilon}\right) \circ \phi^{\epsilon}\right)}{\sqrt{1+\left(\left(\nabla^{f_{\epsilon}} f_{\epsilon}\right) \circ \phi^{\epsilon}\right)^{2}}} \partial_{\epsilon}\left(\left(\nabla^{f_{\epsilon}} f_{\epsilon}\right) \circ \phi^{\epsilon}\right) J_{\phi^{\epsilon}}\right.} \\
& \left.+\sqrt{1+\left(\left(\nabla^{f_{\epsilon}} f_{\epsilon}\right) \circ \phi^{\epsilon}\right)^{2}} \partial_{\epsilon} J_{\phi^{\epsilon}}\right] \mathrm{d} \eta \mathrm{d} \tau .
\end{aligned}
$$

Applying the formula in Proposition 4.1 and the identity $\nabla^{f} \partial_{\epsilon}=\partial_{\epsilon} \nabla^{f}$, one obtains

$$
\partial_{\epsilon}\left(\left(\nabla^{f_{\epsilon}} f_{\epsilon}\right) \circ \phi^{\epsilon}\right)=A_{f}(\epsilon)
$$

and thus formula (14) holds in the smooth case. 
Next, suppose $f=f_{\infty}$ is the limit in $\mathscr{C}_{\mathbf{W}}^{1}$ of a sequence $f_{k} \in \mathscr{C}^{\infty}\left(\mathbf{R}^{2}\right)$, as in Lemma 2.4. Notice that $\nabla^{f_{k}} \phi_{1}^{\epsilon}$ is not zero for $\epsilon$ small enough and $k$ large enough. Indeed, $\left|\nabla^{f_{k}} \phi_{1}^{\epsilon}-\nabla^{f_{\infty}} \phi_{1}^{\epsilon}\right| \leq\left\|f_{k}-f\right\|_{\mathscr{L}^{\infty}(\operatorname{spt} V)}\left\|\partial_{\tau} \phi_{1}^{\epsilon}\right\|_{\mathscr{L} \infty(\operatorname{spt} V)}$ and $\nabla^{f_{\infty}} \phi_{1}^{\epsilon}$ converges to one uniformly on $\mathbf{R}^{2}$ as $\epsilon \rightarrow 0$. Hence, there is an interval $I \subset \mathbf{R}$ centered at zero such that the functions $f_{k, \epsilon}$ as in Proposition 4.1 are well defined for $\epsilon \in I$ and, without loss of generality, for all $k \in \mathbf{N} \cup\{\infty\}$. For $k \in \mathbf{N} \cup\{\infty\}$, define $\gamma_{k}: I \rightarrow \mathbf{R}$ as

$$
\gamma_{k}(\epsilon):=\int_{\omega} \sqrt{1+\left(\nabla^{f_{k, \epsilon}} f_{k, \epsilon}\right)^{2}} \mathrm{~d} \eta \mathrm{d} \tau
$$

Define also the function $\eta: I \rightarrow \mathbf{R}$ as the right-hand side of (14). From Lemma 3.1, it follows that $\left\{A_{f_{k}}\right\}_{k \in \mathbf{N}}$ converges to $A_{f}$ uniformly on $I$. Therefore, we have that $\left\{\gamma_{k}\right\}_{k \in \mathbf{N}}$ and $\left\{\gamma_{k}^{\prime}\right\}_{k \in \mathbf{N}}$ converge to $\gamma$ and $\eta$ uniformly on $I$. We conclude that $\gamma \in$ $\mathscr{C}^{1}(I)$ and $\gamma^{\prime}=\eta$.

In order to evaluate $\gamma^{\prime}(0)$, notice that

$$
\begin{aligned}
\nabla^{f} \phi_{1}^{0} & =1, & \nabla^{f} \phi_{2}^{0} & =f, \\
\left.\nabla^{f} \partial_{\epsilon} \phi_{1}^{\epsilon}\right|_{\epsilon=0} & =\nabla^{f} V_{1}, & \left.\nabla^{f} \partial_{\epsilon} \phi_{2}^{\epsilon}\right|_{\epsilon=0} & =\nabla^{f} V_{2}, \\
\Delta^{f} \phi_{1}^{0} & =0, & \Delta^{f} \phi_{2}^{0} & =\psi, \\
\left.\Delta^{f} \partial_{\epsilon} \phi_{1}^{\epsilon}\right|_{\epsilon=0} & =\Delta^{f} V_{1}, & \left.\Delta^{f} \partial_{\epsilon} \phi_{2}^{\epsilon}\right|_{\epsilon=0} & =\Delta^{f} V_{2} .
\end{aligned}
$$

Therefore

$$
A_{f}(0)=\Delta^{f} V_{2}-2 \psi \nabla^{f} V_{1}-f \Delta^{f} V_{1} .
$$

Moreover, using the facts $\partial_{\tau} \phi_{1}^{0}=\partial_{\eta} \phi_{2}^{0}=0$ and $\partial_{\eta} \phi_{1}^{0}=\partial_{\tau} \phi_{2}^{0}=1$ and that the derivatives $\partial_{\epsilon}, \partial_{\eta}$ and $\partial_{\tau}$ commute, we have

$$
\left.\partial_{\epsilon} J_{\phi^{\epsilon}}\right|_{\epsilon=0}=\partial_{\eta} V_{1}+\partial_{\tau} V_{2} .
$$

Putting all together, we obtain

$$
\begin{aligned}
& \gamma^{\prime}(0)=\int_{\omega} {\left[\frac{\psi}{\sqrt{1+\psi^{2}}}\left(\Delta^{f} V_{2}-2 \psi \cdot \nabla^{f} V_{1}-f \cdot \Delta^{f} V_{1}\right)\right.} \\
&\left.+\sqrt{1+\psi^{2}}\left(\partial_{\eta} V_{1}+\partial_{\tau} V_{2}\right)\right] \mathrm{d} \eta \mathrm{d} \tau
\end{aligned}
$$

Since $\Gamma_{f}$ is an area-minimizing surface, then $\gamma^{\prime}(0)=0$ for all $V_{1}, V_{2} \in \mathscr{C}_{c}^{\infty}\left(\mathbf{R}^{2}\right)$. Since this expression is linear in $V$, then we obtain both conditions (10) and (11).

6.2. Proof of Proposition 6.2. Let $V_{1} \in \mathscr{C}^{\infty}\left(\mathbf{R}^{2}\right)$ and set $V_{2}:=f V_{1} \in$ $\mathscr{C}_{c}^{\infty}\left(\mathbf{R}^{2}\right)$. Then

$$
\begin{aligned}
0= & \int_{\mathbf{R}^{2}}\left[\frac{\psi}{\sqrt{1+\psi^{2}}} \Delta^{f} V_{2}+\sqrt{1+\psi^{2}} \partial_{\tau} V_{2}\right] \mathrm{d} \eta \mathrm{d} \tau \\
= & \int_{\mathbf{R}^{2}}\left[\frac{\psi}{\sqrt{1+\psi^{2}}}\left(\nabla^{f} \psi V_{1}+2 \psi \nabla^{f} V_{1}+f \Delta^{f} V_{1}\right)+\sqrt{1+\psi^{2}}\left(\partial_{\tau} f V_{1}+f \partial_{\tau} V_{1}\right)\right] \mathrm{d} \eta \mathrm{d} \tau \\
= & \int_{\mathbf{R}^{2}}\left[\frac{\psi}{\sqrt{1+\psi^{2}}}\left(2 \psi \nabla^{f} V_{1}+f \Delta^{f} V_{1}\right)+\left(\frac{\psi \nabla^{f} \psi}{\sqrt{1+\psi^{2}}}+\sqrt{1+\psi^{2}} \partial_{\tau} f\right) V_{1}\right. \\
& \left.+\sqrt{1+\psi^{2}}\left(\nabla^{f} V_{1}-\partial_{\eta} V_{1}\right)\right] \mathrm{d} \eta \mathrm{d} \tau
\end{aligned}
$$




$$
\begin{aligned}
&=\int_{\mathbf{R}^{2}} {\left[\frac{\psi}{\sqrt{1+\psi^{2}}}\left(2 \psi \nabla^{f} V_{1}+f \Delta^{f} V_{1}\right)-\sqrt{1+\psi^{2}} \nabla^{f} V_{1}\right.} \\
&\left.+\sqrt{1+\psi^{2}}\left(\nabla^{f} V_{1}-\partial_{\eta} V_{1}\right)\right] \mathrm{d} \eta \mathrm{d} \tau \\
&\left.=\int_{\mathbf{R}^{2}}\left[\frac{\psi}{\sqrt{1+\psi^{2}}}\left(2 \psi \nabla^{f} V_{1}+f \Delta^{f} V_{1}\right)-\sqrt{1+\psi^{2}} \partial_{\eta} V_{1}\right)\right] \mathrm{d} \eta \mathrm{d} \tau .
\end{aligned}
$$

Hence (10) holds true for $V_{1}$ as well.

6.3. Proof of Proposition 6.3. We have for all $V_{2} \in \mathscr{C}_{c}^{\infty}\left(\mathbf{R}^{2}\right)$

$$
\begin{aligned}
& \int_{\mathbf{R}^{2}}\left[\frac{\psi}{\sqrt{1+\psi^{2}}} \nabla^{f} \nabla^{f} V_{2}+\sqrt{1+\psi^{2}} \partial_{\tau} V_{2}\right] \mathrm{d} \eta \mathrm{d} \tau \\
& =-\int_{\mathbf{R}^{2}}\left[\nabla^{f}\left(\frac{\psi}{\sqrt{1+\psi^{2}}}\right) \nabla^{f} V_{2}+\frac{\partial_{\tau} f \psi}{\sqrt{1+\psi^{2}}} \nabla^{f} V_{2}+\partial_{\tau}\left(\sqrt{1+\psi^{2}}\right) V_{2}\right] \mathrm{d} \eta \mathrm{d} \tau \\
& =\int_{\mathbf{R}^{2}}\left[\nabla^{f} \nabla^{f}\left(\frac{\psi}{\sqrt{1+\psi^{2}}}\right) V_{2}+\partial_{\tau} f \nabla^{f}\left(\frac{\psi}{\sqrt{1+\psi^{2}}}\right) V_{2}\right. \\
& +\nabla^{f}\left(\partial_{\tau} f\right) \frac{\psi}{\sqrt{1+\psi^{2}}} V_{2}+\partial_{\tau} f \nabla^{f}\left(\frac{\psi}{\sqrt{1+\psi^{2}}}\right) V_{2}+\left(\partial_{\tau} f\right)^{2} \frac{\psi}{\sqrt{1+\psi^{2}}} V_{2} \\
& \left.-\frac{\psi}{\sqrt{1+\psi^{2}}} \partial_{\tau} \psi V_{2}\right] \mathrm{d} \eta \mathrm{d} \tau .
\end{aligned}
$$

Therefore, using the fact that $\partial_{\tau} \psi=\nabla^{f}\left(\partial_{\tau} f\right)+\left(\partial_{\tau} f\right)^{2}$, we get that (11) is equivalent to

$$
\nabla^{f} \nabla^{f}\left(\frac{\psi}{\sqrt{1+\psi^{2}}}\right)+2 \partial_{\tau} f \cdot \nabla^{f}\left(\frac{\psi}{\sqrt{1+\psi^{2}}}\right)=0
$$

\section{Second contact variation}

Similarly to the previous sections, we set $\psi:=\nabla^{f} f$.

Proposition 7.1. If the intrinsic graph of $f \in \mathscr{C}_{\mathbf{W}}^{1}$ is an area-minimizing surface, then, for all $V_{1}, V_{2} \in \mathscr{C}_{c}^{\infty}\left(\mathbf{R}^{2}\right)$, we have

$$
\begin{aligned}
0 \leq & I I_{f}\left(V_{1}, V_{2}\right):=\int_{\mathbf{R}^{2}}\left[\frac{\left(\Delta^{f} V_{2}-2 \psi \nabla^{f} V_{1}-f \Delta^{f} V_{1}\right)^{2}}{\left(1+\psi^{2}\right)^{\frac{3}{2}}}\right. \\
& +\frac{\psi}{\left(1+\psi^{2}\right)^{\frac{1}{2}}}\left(-4 \Delta^{f} V_{2} \cdot \nabla^{f} V_{1}-2 \nabla^{f} V_{2} \cdot \Delta^{f} V_{1}\right. \\
& \left.\quad+6 f \cdot \nabla^{f} V_{1} \cdot \Delta^{f} V_{1}+6 \psi \cdot\left(\nabla^{f} V_{1}\right)^{2}\right) \\
& +2 \frac{\psi}{\left(1+\psi^{2}\right)^{\frac{1}{2}}}\left(\Delta^{f} V_{2}-2 \psi \nabla^{f} V_{1}-f \Delta^{f} V_{1}\right)\left(\partial_{\eta} V_{1}+\partial_{\tau} V_{2}\right) \\
& \left.+2\left(1+\psi^{2}\right)^{\frac{1}{2}}\left(\partial_{\eta} V_{1} \partial_{\tau} V_{2}-\partial_{\tau} V_{1} \partial_{\eta} V_{2}\right)\right] \mathrm{d} \eta \mathrm{d} \tau .
\end{aligned}
$$


7.1. Proof of Proposition 7.1. Let $\omega \subset \mathbf{R}^{2}$ be an open and bounded set and $V=\left(V_{1}, V_{2}\right): \mathbf{R}^{2} \rightarrow \mathbf{R}^{2}$ a smooth vector field with spt $V \subset \subset \omega$. Let $\phi^{\epsilon}=$ $\left(\phi_{1}^{\epsilon}, \phi_{2}^{\epsilon}\right): \mathbf{R}^{2} \rightarrow \mathbf{R}^{2}$ be a smooth one-parameter family of diffeomorphism such that $\left\{\phi^{\epsilon} \neq \mathrm{Id}\right\} \subset \operatorname{spt} V$ for all $\epsilon>0$ and, for all $p \in \mathbf{R}^{2}$,

$$
\left\{\begin{array}{l}
\phi^{0}(p)=p, \\
\left.\partial_{\epsilon} \phi^{\epsilon}(p)\right|_{\epsilon=0}=V(p) .
\end{array}\right.
$$

Define $W_{i}(p):=\left.\partial_{\epsilon}^{2} \phi_{i}^{\epsilon}(p)\right|_{\epsilon=0}$. Then $W=\left(W_{1}, W_{2}\right): \mathbf{R}^{2} \rightarrow \mathbf{R}^{2}$ is a smooth vector field with spt $W \subset \subset \omega$.

As for the first variation, see Section 6.1, define

$$
\gamma(\epsilon):=\int_{\omega} \sqrt{1+\left(\nabla^{f_{\epsilon}} f_{\epsilon}\right)^{2}} \mathrm{~d} \eta \mathrm{d} \tau
$$

Lemma 7.2. The function $\gamma: I \rightarrow \mathbf{R}$ is twice continuously differentiable and

$$
\begin{aligned}
\gamma^{\prime \prime}(\epsilon)= & \int_{\omega}\left[\frac{A_{f}(\epsilon)^{2}}{\left(1+\left(\nabla^{f_{\epsilon}} f_{\epsilon} \circ \phi^{\epsilon}\right)^{2}\right)^{\frac{3}{2}}} J_{\phi^{\epsilon}}+\frac{\left(\nabla^{f_{\epsilon}} f_{\epsilon} \circ \phi^{\epsilon}\right) B_{f}(\epsilon)}{\left(1+\left(\nabla^{f_{\epsilon}} f_{\epsilon} \circ \phi^{\epsilon}\right)^{2}\right)^{\frac{1}{2}}} J_{\phi^{\epsilon}}\right. \\
& \left.+2 \frac{\left(\nabla^{f_{\epsilon}} f_{\epsilon} \circ \phi^{\epsilon}\right) A_{f}(\epsilon)}{\left(1+\left(\nabla^{f_{\epsilon}} f_{\epsilon} \circ \phi^{\epsilon}\right)^{2}\right)^{\frac{1}{2}}} \partial_{\epsilon} J_{\phi^{\epsilon}}+\left(1+\left(\nabla^{f_{\epsilon}} f_{\epsilon} \circ \phi^{\epsilon}\right)^{2}\right)^{\frac{1}{2}} \partial_{\epsilon}^{2} J_{\phi^{\epsilon}}\right] \mathrm{d} y \mathrm{~d} z,
\end{aligned}
$$

where $A_{f}(\epsilon)$ is defined as in (15) and

$$
\begin{aligned}
B_{f}(\epsilon):= & \frac{\Delta^{f} \partial_{\epsilon}^{2} \phi_{2}^{\epsilon}}{\left(\nabla^{f} \phi_{1}^{\epsilon}\right)^{2}}-2 \frac{\Delta^{f} \partial_{\epsilon} \phi_{2}^{\epsilon} \cdot \nabla^{f} \partial_{\epsilon} \phi_{1}^{\epsilon}}{\left(\nabla^{f} \phi_{1}^{\epsilon}\right)^{3}}-2 \frac{\Delta^{f} \partial_{\epsilon} \phi_{2}^{\epsilon} \cdot \nabla^{f} \partial_{\epsilon} \phi_{1}^{\epsilon}}{\left(\nabla^{f} \phi_{1}^{\epsilon}\right)^{3}} \\
& -2 \frac{\Delta^{f} \phi_{2}^{\epsilon} \cdot \nabla^{f} \partial_{\epsilon}^{2} \phi_{1}^{\epsilon}}{\left(\nabla^{f} \phi_{1}^{\epsilon}\right)^{3}}+6 \frac{\Delta^{f} \phi_{2}^{\epsilon} \cdot\left(\nabla^{f} \partial_{\epsilon} \phi_{1}^{\epsilon}\right)^{2}}{\left(\nabla^{f} \phi_{1}^{\epsilon}\right)^{4}} \\
& -\frac{\nabla^{f} \partial_{\epsilon}^{2} \phi_{2}^{\epsilon} \cdot \Delta^{f} \phi_{1}^{\epsilon}}{\left(\nabla^{f} \phi_{1}^{\epsilon}\right)^{3}}-\frac{\nabla^{f} \partial_{\epsilon} \phi_{2}^{\epsilon} \cdot \Delta^{f} \partial_{\epsilon} \phi_{1}^{\epsilon}}{\left(\nabla^{f} \phi_{1}^{\epsilon}\right)^{3}}+3 \frac{\nabla^{f} \partial_{\epsilon} \phi_{2}^{\epsilon} \cdot \Delta^{f} \phi_{1}^{\epsilon} \cdot \nabla^{f} \partial_{\epsilon} \phi_{1}^{\epsilon}}{\left(\nabla^{f} \phi_{1}^{\epsilon}\right)^{4}} \\
& +3 \frac{\nabla^{f} \partial_{\epsilon} \phi_{2}^{\epsilon} \cdot \nabla^{f} \partial_{\epsilon} \phi_{1}^{\epsilon} \cdot \Delta^{f} \phi_{1}^{\epsilon}}{\left(\nabla^{f} \phi_{1}^{\epsilon}\right)^{4}}+3 \frac{\nabla^{f} \phi_{2}^{\epsilon} \cdot \nabla^{f} \partial_{\epsilon}^{2} \phi_{1}^{\epsilon} \cdot \Delta^{f} \phi_{1}^{\epsilon}}{\left(\nabla^{f} \phi_{1}^{\epsilon}\right)^{4}} \\
& +3 \frac{\nabla^{f} \phi_{2}^{\epsilon} \cdot \nabla^{f} \partial_{\epsilon} \phi_{1}^{\epsilon} \cdot \Delta^{f} \partial_{\epsilon} \phi_{1}^{\epsilon}}{\left(\nabla^{f} \phi_{1}^{\epsilon}\right)^{4}}-12 \frac{\nabla^{f} \phi_{2}^{\epsilon} \cdot\left(\nabla^{f} \partial_{\epsilon} \phi_{1}^{\epsilon}\right)^{2} \cdot \Delta^{f} \phi_{1}^{\epsilon}}{\left(\nabla^{f} \phi_{1}^{\epsilon}\right)^{5}} \\
& -\frac{\nabla^{f} \partial_{\epsilon} \phi_{2}^{\epsilon} \cdot \Delta^{f} \partial_{\epsilon} \phi_{1}^{\epsilon}}{\left(\nabla^{f} \phi_{1}^{\epsilon}\right)^{3}}-\frac{\nabla^{f} \phi_{2}^{\epsilon} \cdot \Delta^{f} \partial_{\epsilon}^{2} \phi_{1}^{\epsilon}}{\left(\nabla^{f} \phi_{1}^{\epsilon}\right)^{3}}+3 \frac{\nabla^{f} \phi_{2}^{\epsilon} \cdot \Delta^{f} \partial_{\epsilon} \phi_{1}^{\epsilon} \cdot \nabla^{f} \partial_{\epsilon} \phi_{1}^{\epsilon}}{\left(\nabla^{f} \phi_{1}^{\epsilon}\right)^{4}}
\end{aligned}
$$

Proof of Lemma 7.2. This lemma is a continuation of Lemma 6.4. First, suppose $f \in \mathscr{C}^{\infty}\left(\mathbf{R}^{2}\right)$. Then, the function $\gamma$ is smooth and its second derivative is

$$
\begin{aligned}
\gamma^{\prime \prime}(\epsilon)= & \int_{\omega}\left[\frac{\left(\partial_{\epsilon}\left(\nabla^{f_{\epsilon}} f_{\epsilon} \circ \phi^{\epsilon}\right)\right)^{2}}{\left(1+\left(\nabla^{f_{\epsilon}} f_{\epsilon} \circ \phi^{\epsilon}\right)^{2}\right)^{\frac{3}{2}}} J_{\phi^{\epsilon}}+\frac{\left(\nabla^{f_{\epsilon}} f_{\epsilon} \circ \phi^{\epsilon}\right) \partial_{\epsilon}^{2}\left(\nabla^{f_{\epsilon}} f_{\epsilon} \circ \phi^{\epsilon}\right)}{\left(1+\left(\nabla^{f_{\epsilon}} f_{\epsilon} \circ \phi^{\epsilon}\right)^{2}\right)^{\frac{1}{2}}} J_{\phi^{\epsilon}}\right. \\
& \left.+2 \frac{\left(\nabla^{f_{\epsilon}} f_{\epsilon} \circ \phi^{\epsilon}\right) \partial_{\epsilon}\left(\nabla^{f_{\epsilon}} f_{\epsilon} \circ \phi^{\epsilon}\right)}{\left(1+\left(\nabla^{f_{\epsilon}} f_{\epsilon} \circ \phi^{\epsilon}\right)^{2}\right)^{\frac{1}{2}}} \partial_{\epsilon} J_{\phi^{\epsilon}}+\left(1+\left(\nabla^{f_{\epsilon}} f_{\epsilon} \circ \phi^{\epsilon}\right)^{2}\right)^{\frac{1}{2}} \partial_{\epsilon}^{2} J_{\phi^{\epsilon}}\right] \mathrm{d} y \mathrm{~d} z .
\end{aligned}
$$

One checks by direct computation that

$$
\begin{aligned}
& \partial_{\epsilon}\left(\nabla^{f_{\epsilon}} f_{\epsilon} \circ \phi^{\epsilon}\right)=A_{f}(\epsilon), \\
& \partial_{\epsilon}^{2}\left(\nabla^{f_{\epsilon}} f_{\epsilon} \circ \phi^{\epsilon}\right)=B_{f}(\epsilon),
\end{aligned}
$$

thus (17) is proven in the smooth case. 
Next, suppose $f=f_{\infty}$ is the limit in $\mathscr{C}_{\mathbf{W}}^{1}$ of a sequence $f_{k} \in \mathscr{C}^{\infty}\left(\mathbf{R}^{2}\right)$, as in Lemma 2.4. Define $f_{k, \epsilon}$ and $I \subset \mathbf{R}$ and $\gamma_{k}: I \rightarrow \mathbf{R}$ as in the proof of Lemma 6.4. Define also $\eta: I \rightarrow \mathbf{R}$ as the right-hand side of (17). By Lemma 3.1, $\left\{A_{f_{k}}\right\}_{k \in \mathbf{N}}$ and $\left\{B_{f_{k}}\right\}_{k \in \mathbf{N}}$ converge to $A_{f}$ and $B_{f}$ uniformly on $I$. Therefore, we have that the convergences $\gamma_{k} \rightarrow \gamma$ and $\gamma_{k}^{\prime} \rightarrow \gamma^{\prime}$ and $\gamma_{k}^{\prime \prime} \rightarrow \eta$ are uniform on $I$. We conclude that $\gamma \in \mathscr{C}^{2}(I)$ and $\gamma^{\prime \prime}=\eta$.

Next, one can directly check that

$$
\begin{aligned}
\gamma^{\prime \prime}(0)=\int_{\omega}\left[\frac{\left(\Delta^{f} V_{2}-2 \psi \nabla^{f} V_{1}-f \Delta^{f} V_{1}\right)^{2}}{\left(1+\psi^{2}\right)^{\frac{3}{2}}}+\frac{\psi}{\left(1+\psi^{2}\right)^{\frac{1}{2}}}\left(\Delta^{f} W_{2}-f \cdot \Delta^{f} W_{1}\right.\right. \\
\quad-2 \psi \cdot \nabla^{f} W_{1}-4 \Delta^{f} V_{2} \cdot \nabla^{f} V_{1}-2 \nabla^{f} V_{2} \cdot \Delta^{f} V_{1} \\
\left.\quad+6 f \cdot \nabla^{f} V_{1} \cdot \Delta^{f} V_{1}+6 \psi \cdot\left(\nabla^{f} V_{1}\right)^{2}\right) \\
+2 \frac{\psi}{\left(1+\psi^{2}\right)^{\frac{1}{2}}}\left(\Delta^{f} V_{2}-2 \psi \nabla^{f} V_{1}-f \Delta^{f} V_{1}\right)\left(\partial_{\eta} V_{1}+\partial_{\tau} V_{2}\right) \\
\left.+\left(1+\psi^{2}\right)^{\frac{1}{2}}\left(\partial_{\eta} W_{1}+\partial_{\tau} W_{2}+2\left(\partial_{\eta} V_{1} \partial_{\tau} V_{2}-\partial_{\tau} V_{1} \partial_{\eta} V_{2}\right)\right)\right] \mathrm{d} \eta \mathrm{d} \tau
\end{aligned}
$$

Finally, if $\Gamma_{f}$ is an area-minimizing surface, then $\gamma^{\prime}(0)=0$ and $\gamma^{\prime \prime}(0) \geq 0$. Notice that the terms containing $W_{1}$ and $W_{2}$ in the expression of $\gamma^{\prime \prime}(0)$ are zero because $\gamma^{\prime}(0)=0$. So, the second variation formula (16) is proven.

\section{Contact variations in the case $\Delta^{f} f=0$}

In this final section we prove our main result. We show that there is a quite large class of functions in $\mathscr{C}_{\mathbf{W}}^{1}$ that satisfy both conditions on the first and second contact variation. Since we know that the only intrinsic graphs of smooth functions that are area minimizers are the vertical planes, our result shows that variations along contact diffeomorphisms are not selective enough.

As usual, we set $\psi:=\nabla^{f} f$.

Lemma 8.1. Let $f \in \mathscr{C}^{\infty}\left(\mathbf{R}^{2}\right)$ be such that $\Delta^{f} f=0$. Then

$$
\begin{aligned}
I I_{f}\left(V_{1}, V_{2}\right)= & \int_{\mathbf{R}^{2}}\left[\frac{\left(\Delta^{f} V_{2}-2 \psi \nabla^{f} V_{1}-f \Delta^{f} V_{1}\right)^{2}}{\left(1+\psi^{2}\right)^{\frac{3}{2}}}\right. \\
& \left.+\partial_{\tau}\left(\frac{\psi}{\left(1+\psi^{2}\right)^{\frac{1}{2}}}\right)\left(\nabla^{f} V_{2}-\nabla^{f}\left(f V_{1}\right)\right)^{2}\right] \mathrm{d} \eta \mathrm{d} \tau .
\end{aligned}
$$

The proof is very technical and it is postponed to the last section below.

Theorem 8.2. Let $f \in \mathscr{C}_{\mathbf{W}}^{1}$ be such that $\Delta^{f} f=0$ in weak Lagrangian sense. Then both equalities (10) and (11) and also the inequality (16) are satisfied for all $V_{1}, V_{2} \in \mathscr{C}_{c}^{\infty}\left(\mathbf{R}^{2}\right)$.

Proof. We first prove that both equalities (10) and (11) are satisfied. Let $\left\{f_{k}\right\}_{k \in \mathbf{N}} \subset \mathscr{C}^{\infty}\left(\mathbf{R}^{2}\right)$ be a sequence converging to $f$ in $\mathscr{C}_{\mathbf{W}}^{1}$ and such that $\Delta^{f_{k}} f_{k}=0$, as in Lemma 3.8. Fix $V_{1}, V_{2} \in \mathscr{C}_{c}^{\infty}\left(\mathbf{R}^{2}\right)$. Then (12) and (10) are satisfied by all $f_{k}$ thanks to Propositions 6.2 and 6.3. Passing to the limit $k \rightarrow \infty$, we prove that $f$ satisfies them too.

Now, we prove that the inequality (16) holds true. If $f \in \mathscr{C}^{\infty}\left(\mathbf{R}^{2}\right)$, then we can apply Lemma 8.1, where $\partial_{\tau}\left(\frac{\psi}{\left(1+\psi^{2}\right)^{\frac{1}{2}}}\right)=\frac{\partial_{\tau} \psi}{\left(1+\psi^{2}\right)^{\frac{3}{2}}} \geq 0$ because of Lemma 3.5. So, (16) 
is proven for $f$ smooth. For $f \in \mathscr{C}_{\mathbf{W}}^{1}$, let $\left\{f_{k}\right\}_{k \in \mathbf{N}} \subset \mathscr{C}^{\infty}\left(\mathbf{R}^{2}\right)$ as in Lemma 3.8. From Lemma 3.1 follows that, for fixed $V_{1}, V_{2} \in \mathscr{C}_{c}^{\infty}\left(\mathbf{R}^{2}\right)$, it holds

$$
\lim _{k \rightarrow \infty} I I_{f_{k}}\left(V_{1}, V_{2}\right)=I I_{f}\left(V_{1}, V_{2}\right),
$$

thus $I I_{f}\left(V_{1}, V_{2}\right) \geq 0$.

8.1. Proof of Lemma 8.1. The proof of this lemma is just a computation, but quite elaborate. For making the formulas more readable, we decided to drop the sign of integral along the proof. In other words, all equalities in this section are meant as equalities of integrals on $\mathbf{R}^{2}$. We will constantly use the formulas listed in Appendix A together with $\nabla^{f} \psi=0$.

Before of all, we reorganise the integral in (16):

$$
\begin{aligned}
& \frac{\left(\Delta^{f} V_{2}-2 \psi \nabla^{f} V_{1}-f \Delta^{f} V_{1}\right)^{2}}{\left(1+\psi^{2}\right)^{\frac{3}{2}}} \\
& +\frac{\psi}{\left(1+\psi^{2}\right)^{\frac{1}{2}}}\left(+6 f \cdot \nabla^{f} V_{1} \cdot \Delta^{f} V_{1}+6 \psi \cdot\left(\nabla^{f} V_{1}\right)^{2}\right) \\
& +2 \frac{\psi}{\left(1+\psi^{2}\right)^{\frac{1}{2}}}\left(-2 \psi \nabla^{f} V_{1}-f \Delta^{f} V_{1}\right) \partial_{\eta} V_{1} \\
& +2 \frac{\psi}{\left(1+\psi^{2}\right)^{\frac{1}{2}}} \Delta^{f} V_{2} \partial_{\tau} V_{2} \\
& +\frac{\psi}{\left(1+\psi^{2}\right)^{\frac{1}{2}}}\left(-4 \Delta^{f} V_{2} \cdot \nabla^{f} V_{1}-2 \nabla^{f} V_{2} \cdot \Delta^{f} V_{1}\right) \\
& +2 \frac{\psi}{\left(1+\psi^{2}\right)^{\frac{1}{2}}}\left(\Delta^{f} V_{2} \partial_{\eta} V_{1}+\left(-2 \psi \nabla^{f} V_{1}-f \Delta^{f} V_{1}\right) \partial_{\tau} V_{2}\right) \\
& +2\left(1+\psi^{2}\right)^{\frac{1}{2}}\left(\partial_{\eta} V_{1} \partial_{\tau} V_{2}-\partial_{\tau} V_{1} \partial_{\eta} V_{2}\right) .
\end{aligned}
$$

In the following lemmas we will study (b) + (C), (d) and (e) $+(1)+$ (g) separately in order to obtain the expansion of the square in the second term of the integral in Lemma 8.1.

\section{Lemma 8.3.}

$$
\text { (b) }+ \text { (C) }=\partial_{\tau}\left(\frac{\psi}{\left(1+\psi^{2}\right)^{\frac{1}{2}}}\right)\left(\nabla^{f}\left(f V_{1}\right)\right)^{2} \text {. }
$$

Proof of Lemma 8.3.

$$
\begin{aligned}
\text { (b) } & =\frac{\psi}{\left(1+\psi^{2}\right)^{\frac{1}{2}}}\left(6 f \nabla^{f} V_{1} \Delta^{f} V_{1}+6 \psi\left(\nabla^{f} V_{1}\right)^{2}\right) \\
& =\frac{\psi}{\left(1+\psi^{2}\right)^{\frac{1}{2}}}\left(3 f \nabla^{f}\left(\nabla^{f} V_{1}\right)^{2}+6 \psi\left(\nabla^{f} V_{1}\right)^{2}\right) \\
& =3 \frac{\psi}{\left(1+\psi^{2}\right)^{\frac{1}{2}}}\left(\nabla^{f}\left(f\left(\nabla^{f} V_{1}\right)^{2}\right)+\psi\left(\nabla^{f} V_{1}\right)^{2}\right) \\
& =-3 \frac{\psi}{\left(1+\psi^{2}\right)^{\frac{1}{2}}} f \partial_{\tau} f\left(\nabla^{f} V_{1}\right)^{2}+3 \frac{\psi^{2}}{\left(1+\psi^{2}\right)^{\frac{1}{2}}}\left(\nabla^{f} V_{1}\right)^{2} \\
& =-\frac{3}{2} \frac{\psi}{\left(1+\psi^{2}\right)^{\frac{1}{2}}} \partial_{\tau}\left(f^{2}\right)\left(\nabla^{f} V_{1}\right)^{2}+3 \frac{\psi^{2}}{\left(1+\psi^{2}\right)^{\frac{1}{2}}}\left(\nabla^{f} V_{1}\right)^{2} .
\end{aligned}
$$




$$
\begin{aligned}
\text { C }= & -2 \frac{\psi}{\left(1+\psi^{2}\right)^{\frac{1}{2}}}\left(2 \psi \nabla^{f} V_{1}+f \Delta^{f} V_{1}\right) \partial_{\eta} V_{1} \\
= & -2 \frac{\psi}{\left(1+\psi^{2}\right)^{\frac{1}{2}}}\left(2 \psi \nabla^{f} V_{1}+f \Delta^{f} V_{1}\right)\left(\nabla^{f} V_{1}-f \partial_{\tau} V_{1}\right) \\
= & -4 \frac{\psi^{2}}{\left(1+\psi^{2}\right)^{\frac{1}{2}}}\left(\nabla^{f} V_{1}\right)^{2}+4 \frac{\psi^{2}}{\left(1+\psi^{2}\right)^{\frac{1}{2}}} f \nabla^{f} V_{1} \partial_{\tau} V_{1} \\
& -2 \frac{\psi}{\left(1+\psi^{2}\right)^{\frac{1}{2}}} f \Delta^{f} V_{1} \nabla^{f} V_{1}+2 \frac{\psi}{\left(1+\psi^{2}\right)^{\frac{1}{2}}} f^{2} \Delta^{f} V_{1} \partial_{\tau} V_{1} .
\end{aligned}
$$

We have two particular terms in this expression:

$$
\begin{aligned}
@ & :=4 \frac{\psi^{2}}{\left(1+\psi^{2}\right)^{\frac{1}{2}}} f \nabla^{f} V_{1} \partial_{\tau} V_{1}+2 \frac{\psi}{\left(1+\psi^{2}\right)^{\frac{1}{2}}} f^{2} \Delta^{f} V_{1} \partial_{\tau} V_{1} \\
& =2 \frac{\psi}{\left(1+\psi^{2}\right)^{\frac{1}{2}}} \nabla^{f}\left(f^{2} \nabla^{f} V_{1}\right) \partial_{\tau} V_{1} \\
& =-2 \frac{\psi}{\left(1+\psi^{2}\right)^{\frac{1}{2}}}\left(f^{2} \nabla^{f} V_{1}\right) \nabla^{f} \partial_{\tau} V_{1}-2 \partial_{\tau} f \frac{\psi}{\left(1+\psi^{2}\right)^{\frac{1}{2}}}\left(f^{2} \nabla^{f} V_{1}\right) \partial_{\tau} V_{1} \\
& =-2 \frac{\psi}{\left(1+\psi^{2}\right)^{\frac{1}{2}}} f^{2} \nabla^{f} V_{1}\left(\nabla^{f} \partial_{\tau} V_{1}+\partial_{\tau} f \partial_{\tau} V_{1}\right) \\
& =-2 \frac{\psi}{\left(1+\psi^{2}\right)^{\frac{1}{2}}} f^{2} \nabla^{f} V_{1} \partial_{\tau} \nabla^{f} V_{1}=-\frac{\psi}{\left(1+\psi^{2}\right)^{\frac{1}{2}}} f^{2} \partial_{\tau}\left(\nabla^{f} V_{1}\right)^{2} \\
& =\partial_{\tau}\left(\frac{\psi}{\left(1+\psi^{2}\right)^{\frac{1}{2}}}\right) f^{2}\left(\nabla^{f} V_{1}\right)^{2}+\frac{\psi}{\left(1+\psi^{2}\right)^{\frac{1}{2}}} \partial_{\tau}\left(f^{2}\right)\left(\nabla^{f} V_{1}\right)^{2}
\end{aligned}
$$

and

$$
\begin{aligned}
(3) & :=-2 \frac{\psi}{\left(1+\psi^{2}\right)^{\frac{1}{2}}} f \Delta^{f} V_{1} \nabla^{f} V_{1} \\
& =-\frac{\psi}{\left(1+\psi^{2}\right)^{\frac{1}{2}}} f \nabla^{f}\left(\nabla^{f} V_{1}\right)^{2}=\frac{\psi^{2}}{\left(1+\psi^{2}\right)^{\frac{1}{2}}}\left(\nabla^{f} V_{1}\right)^{2}+\frac{\psi}{\left(1+\psi^{2}\right)^{\frac{1}{2}}} f \partial_{\tau} f\left(\nabla^{f} V_{1}\right)^{2} \\
& =\frac{\psi^{2}}{\left(1+\psi^{2}\right)^{\frac{1}{2}}}\left(\nabla^{f} V_{1}\right)^{2}+\frac{\psi}{\left(1+\psi^{2}\right)^{\frac{1}{2}}} \frac{\partial_{\tau}\left(f^{2}\right)}{2}\left(\nabla^{f} V_{1}\right)^{2} .
\end{aligned}
$$

Therefore

$$
\begin{aligned}
(C)= & -4 \frac{\psi^{2}}{\left(1+\psi^{2}\right)^{\frac{1}{2}}}\left(\nabla^{f} V_{1}\right)^{2}+@+ß \\
= & -3 \frac{\psi^{2}}{\left(1+\psi^{2}\right)^{\frac{1}{2}}}\left(\nabla^{f} V_{1}\right)^{2}+\partial_{\tau}\left(\frac{\psi}{\left(1+\psi^{2}\right)^{\frac{1}{2}}}\right) f^{2}\left(\nabla^{f} V_{1}\right)^{2} \\
& +\frac{3}{2} \frac{\psi}{\left(1+\psi^{2}\right)^{\frac{1}{2}}} \partial_{\tau}\left(f^{2}\right)\left(\nabla^{f} V_{1}\right)^{2}
\end{aligned}
$$


Putting this together,

$$
\begin{aligned}
\text { (b) }+ \text { C } & =\partial_{\tau}\left(\frac{\psi}{\left(1+\psi^{2}\right)^{\frac{1}{2}}}\right) f^{2}\left(\nabla^{f} V_{1}\right)^{2} \\
& =\partial_{\tau}\left(\frac{\psi}{\left(1+\psi^{2}\right)^{\frac{1}{2}}}\right)\left(\nabla^{f}\left(f V_{1}\right)-\psi V_{1}\right)^{2} \\
& =\partial_{\tau}\left(\frac{\psi}{\left(1+\psi^{2}\right)^{\frac{1}{2}}}\right)\left(\left(\nabla^{f}\left(f V_{1}\right)\right)^{2}+\left(\psi V_{1}\right)^{2}-2 \nabla^{f}\left(f V_{1}\right) \psi V_{1}\right) \\
& =\partial_{\tau}\left(\frac{\psi}{\left(1+\psi^{2}\right)^{\frac{1}{2}}}\right)\left(\left(\nabla^{f}\left(f V_{1}\right)\right)^{2}-\left(\psi V_{1}\right)^{2}-f \nabla^{f}\left(\psi V_{1}^{2}\right)\right) \\
& \stackrel{(*)}{=} \partial_{\tau}\left(\frac{\psi}{\left(1+\psi^{2}\right)^{\frac{1}{2}}}\right)\left(\left(\nabla^{f}\left(f V_{1}\right)\right)^{2}-\left(\psi V_{1}\right)^{2}+\nabla^{f} f \psi V_{1}^{2}\right) \\
& =\partial_{\tau}\left(\frac{\psi}{\left(1+\psi^{2}\right)^{\frac{1}{2}}}\right)\left(\nabla^{f}\left(f V_{1}\right)\right)^{2} .
\end{aligned}
$$

In $(*)$ we used formula (18).

\section{Lemma 8.4.}

$$
\text { (d) }=\partial_{\tau}\left(\frac{\psi}{\left(1+\psi^{2}\right)^{\frac{1}{2}}}\right)\left(\nabla^{f} V_{2}\right)^{2} \text {. }
$$

Proof of Lemma 8.4.

$$
\text { (d) } \begin{aligned}
& =2 \frac{\psi}{\left(1+\psi^{2}\right)^{\frac{1}{2}}} \Delta^{f} V_{2} \partial_{\tau} V_{2} \stackrel{(*)}{=}-2 \frac{\psi}{\left(1+\psi^{2}\right)^{\frac{1}{2}}} \nabla^{f} V_{2} \partial_{\tau} \nabla^{f} V_{2} \\
& =-\frac{\psi}{\left(1+\psi^{2}\right)^{\frac{1}{2}}} \partial_{\tau}\left(\nabla^{f} V_{2}\right)^{2}=\partial_{\tau}\left(\frac{\psi}{\left(1+\psi^{2}\right)^{\frac{1}{2}}}\right)\left(\nabla^{f} V_{2}\right)^{2} .
\end{aligned}
$$

In $(*)$ we used formula (18).

\section{Lemma 8.5.}

$$
\left(\mathrm{e}+\mathrm{f}+\mathrm{g}=-2 \partial_{\tau}\left(\frac{\psi}{\left(1+\psi^{2}\right)^{\frac{1}{2}}}\right) \nabla^{f}\left(f V_{1}\right) \nabla^{f} V_{2}\right. \text {. }
$$

Proof.

$$
\begin{aligned}
\mathrm{g} & =2\left(1+\psi^{2}\right)^{\frac{1}{2}}\left(\partial_{\eta} V_{1} \partial_{\tau} V_{2}-\partial_{\tau} V_{1} \partial_{\eta} V_{2}\right) \\
& =2\left(1+\psi^{2}\right)^{\frac{1}{2}}\left(\left(\nabla^{f} V_{1}-f \partial_{\tau} V_{1}\right) \partial_{\tau} V_{2}-\partial_{\tau} V_{1}\left(\nabla^{f} V_{2}-f \partial_{\tau} V_{2}\right)\right) \\
& =2\left(1+\psi^{2}\right)^{\frac{1}{2}}\left(\nabla^{f} V_{1} \partial_{\tau} V_{2}-\partial_{\tau} V_{1} \nabla^{f} V_{2}\right) . \\
\mathbb{f} & =2 \frac{\psi}{\left(1+\psi^{2}\right)^{\frac{1}{2}}}\left(\Delta^{f} V_{2} \partial_{\eta} V_{1}+\left(-2 \psi \nabla^{f} V_{1}-f \Delta^{f} V_{1}\right) \partial_{\tau} V_{2}\right) \\
& =2 \frac{\psi}{\left(1+\psi^{2}\right)^{\frac{1}{2}}}\left(\Delta^{f} V_{2}\left(\nabla^{f} V_{1}-f \partial_{\tau} V_{1}\right)-2 \psi \nabla^{f} V_{1} \partial_{\tau} V_{2}-f \Delta^{f} V_{1} \partial_{\tau} V_{2}\right) \\
& =2 \frac{\psi}{\left(1+\psi^{2}\right)^{\frac{1}{2}}}\left(-f \partial_{\tau} V_{1} \Delta^{f} V_{2}-f \partial_{\tau} V_{2} \Delta^{f} V_{1}+\Delta^{f} V_{2} \nabla^{f} V_{1}-2 \psi \nabla^{f} V_{1} \partial_{\tau} V_{2}\right)
\end{aligned}
$$




$$
\begin{gathered}
\stackrel{(*)}{=} 2 \frac{\psi}{\left(1+\psi^{2}\right)^{\frac{1}{2}}}\left(\psi \partial_{\tau} V_{1} \nabla^{f} V_{2}+f \partial_{\tau}\left(\nabla^{f} V_{1}\right) \nabla^{f} V_{2}+\psi \partial_{\tau} V_{2} \nabla^{f} V_{1}+\right. \\
\left.+f \partial_{\tau}\left(\nabla^{f} V_{2}\right) \nabla^{f} V_{1}+\Delta^{f} V_{2} \nabla^{f} V_{1}-2 \psi \nabla^{f} V_{1} \partial_{\tau} V_{2}\right) \\
=2 \frac{\psi}{\left(1+\psi^{2}\right)^{\frac{1}{2}}}\left(\psi \partial_{\tau} V_{1} \nabla^{f} V_{2}+f \partial_{\tau}\left(\nabla^{f} V_{1}\right) \nabla^{f} V_{2}+\right. \\
\left.+f \partial_{\tau}\left(\nabla^{f} V_{2}\right) \nabla^{f} V_{1}+\Delta^{f} V_{2} \nabla^{f} V_{1}-\psi \nabla^{f} V_{1} \partial_{\tau} V_{2}\right) .
\end{gathered}
$$

In $(*)$ we used formula (18).

$$
\begin{aligned}
& (\mathfrak{f}+\mathrm{g})=2 \frac{\left(1+\psi^{2}\right)}{\left(1+\psi^{2}\right)^{\frac{1}{2}}}\left(\nabla^{f} V_{1} \partial_{\tau} V_{2}-\partial_{\tau} V_{1} \nabla^{f} V_{2}\right) \\
& +2 \frac{\psi}{\left(1+\psi^{2}\right)^{\frac{1}{2}}}\left(\psi \partial_{\tau} V_{1} \nabla^{f} V_{2}+f \partial_{\tau}\left(\nabla^{f} V_{1}\right) \nabla^{f} V_{2}\right. \\
& \left.+f \partial_{\tau}\left(\nabla^{f} V_{2}\right) \nabla^{f} V_{1}+\Delta^{f} V_{2} \nabla^{f} V_{1}-\psi \nabla^{f} V_{1} \partial_{\tau} V_{2}\right) \\
& =2 \frac{1}{\left(1+\psi^{2}\right)^{\frac{1}{2}}}\left(\nabla^{f} V_{1} \partial_{\tau} V_{2}-\partial_{\tau} V_{1} \nabla^{f} V_{2}\right) \\
& +2 \frac{\psi}{\left(1+\psi^{2}\right)^{\frac{1}{2}}}\left(f \partial_{\tau}\left(\nabla^{f} V_{1} \nabla^{f} V_{2}\right)+\Delta^{f} V_{2} \nabla^{f} V_{1}\right) . \\
& \text { ( }+(1)+\text { g }=\frac{\psi}{\left(1+\psi^{2}\right)^{\frac{1}{2}}}\left(-4 \Delta^{f} V_{2} \nabla^{f} V_{1}-2 \nabla^{f} V_{2} \Delta^{f} V_{1}\right)+(\mathrm{f}+\mathrm{g} \\
& =2 \frac{\psi}{\left(1+\psi^{2}\right)^{\frac{1}{2}}}\left(-\Delta^{f} V_{2} \nabla^{f} V_{1}-\nabla^{f} V_{2} \Delta^{f} V_{1}+f \partial_{\tau}\left(\nabla^{f} V_{1} \nabla^{f} V_{2}\right)\right) \\
& +2 \frac{1}{\left(1+\psi^{2}\right)^{\frac{1}{2}}}\left(\nabla^{f} V_{1} \partial_{\tau} V_{2}-\partial_{\tau} V_{1} \nabla^{f} V_{2}\right) \\
& =2 \frac{\psi}{\left(1+\psi^{2}\right)^{\frac{1}{2}}}\left(-\nabla^{f}\left(\nabla^{f} V_{2} \nabla^{f} V_{1}\right)+f \partial_{\tau}\left(\nabla^{f} V_{1} \nabla^{f} V_{2}\right)\right) \\
& +2 \frac{1}{\left(1+\psi^{2}\right)^{\frac{1}{2}}}\left(\nabla^{f} V_{1} \partial_{\tau} V_{2}-\partial_{\tau} V_{1} \nabla^{f} V_{2}\right) \\
& =2 \frac{\psi}{\left(1+\psi^{2}\right)^{\frac{1}{2}}} \partial_{\tau} f \nabla^{f} V_{2} \nabla^{f} V_{1}-2 \partial_{\tau}\left(\frac{\psi}{\left(1+\psi^{2}\right)^{\frac{1}{2}}}\right) f \nabla^{f} V_{1} \nabla^{f} V_{2} \\
& -2 \frac{\psi}{\left(1+\psi^{2}\right)^{\frac{1}{2}}} \partial_{\tau} f \nabla^{f} V_{1} \nabla^{f} V_{2}+2 \frac{1}{\left(1+\psi^{2}\right)^{\frac{1}{2}}}\left(\nabla^{f} V_{1} \partial_{\tau} V_{2}-\partial_{\tau} V_{1} \nabla^{f} V_{2}\right) \\
& =-2 \partial_{\tau}\left(\frac{\psi}{\left(1+\psi^{2}\right)^{\frac{1}{2}}}\right) f \nabla^{f} V_{1} \nabla^{f} V_{2} \\
& +2 \frac{1}{\left(1+\psi^{2}\right)^{\frac{1}{2}}}\left(\nabla^{f} V_{1} \partial_{\tau} V_{2}-\partial_{\tau} V_{1} \nabla^{f} V_{2}\right)
\end{aligned}
$$


In particular, we have

$$
\begin{aligned}
& \frac{1}{\left(1+\psi^{2}\right)^{\frac{1}{2}}}\left(\nabla^{f} V_{1} \partial_{\tau} V_{2}-\partial_{\tau} V_{1} \nabla^{f} V_{2}\right) \\
& =-\frac{1}{\left(1+\psi^{2}\right)^{\frac{1}{2}}} \partial_{\tau} \nabla^{f} V_{2} V_{1}+\partial_{\tau}\left(\frac{1}{\left(1+\psi^{2}\right)^{\frac{1}{2}}}\right) \nabla^{f} V_{2} V_{1}+\frac{1}{\left(1+\psi^{2}\right)^{\frac{1}{2}}} \partial_{\tau} \nabla^{f} V_{2} V_{1} \\
& =\partial_{\tau}\left(\frac{1}{\left(1+\psi^{2}\right)^{\frac{1}{2}}}\right) \nabla^{f} V_{2} V_{1}
\end{aligned}
$$

and

$$
\partial_{\tau}\left(\frac{1}{\left(1+\psi^{2}\right)^{\frac{1}{2}}}\right)=-\frac{1}{\left(1+\psi^{2}\right)^{\frac{3}{2}}} \psi \partial_{\tau} \psi=-\psi \partial_{\tau}\left(\frac{\psi}{\left(1+\psi^{2}\right)^{\frac{1}{2}}}\right) .
$$

Therefore

$$
\begin{aligned}
& \text { e }+ \text { f }+ \text { (g) } \\
& =-2 \partial_{\tau}\left(\frac{\psi}{\left(1+\psi^{2}\right)^{\frac{1}{2}}}\right) f \nabla^{f} V_{1} \nabla^{f} V_{2}+2 \frac{1}{\left(1+\psi^{2}\right)^{\frac{1}{2}}}\left(\nabla^{f} V_{1} \partial_{\tau} V_{2}-\partial_{\tau} V_{1} \nabla^{f} V_{2}\right) \\
& =-2 \partial_{\tau}\left(\frac{\psi}{\left(1+\psi^{2}\right)^{\frac{1}{2}}}\right) f \nabla^{f} V_{1} \nabla^{f} V_{2}-2 \psi \partial_{\tau}\left(\frac{\psi}{\left(1+\psi^{2}\right)^{\frac{1}{2}}}\right) \nabla^{f} V_{2} V_{1} \\
& =-2 \partial_{\tau}\left(\frac{\psi}{\left(1+\psi^{2}\right)^{\frac{1}{2}}}\right) \nabla^{f}\left(f V_{1}\right) \nabla^{f} V_{2} .
\end{aligned}
$$

\section{Appendix A. Useful formulas}

In the case $f \in \mathscr{C}^{\infty}\left(\mathbf{R}^{2}\right)$, the adjoint operator of $\nabla^{f}$ is

$$
\left(\nabla^{f}\right)^{*}=-\nabla^{f}-\partial_{\tau} f
$$

i.e., if $A, B \in \mathscr{C}^{\infty}\left(\mathbf{R}^{2}\right)$ and one of them has compact support, then

$$
\int_{\mathbf{R}^{2}} A \cdot \nabla^{f} B \mathrm{~d} \eta \mathrm{d} \tau=-\int_{\mathbf{R}^{2}}\left[\nabla^{f} A \cdot B+\partial_{\tau} f \cdot A \cdot B\right] \mathrm{d} \eta \mathrm{d} \tau
$$

Notice that, if $f$ is smooth, the following holds:

$$
\begin{aligned}
\partial_{\eta} & =\nabla^{f}-f \partial_{\tau}, \\
\partial_{\tau} \nabla^{f} & =\nabla^{f} \partial_{\tau}+\partial_{\tau} f \partial_{\tau} .
\end{aligned}
$$

If $A, B, C \in \mathscr{C}^{\infty}\left(\mathbf{R}^{2}\right)$ and one of them has compact support, then

$$
\int_{\mathbf{R}^{2}} A \cdot \partial_{\tau} B \cdot \nabla^{f} C \mathrm{~d} \eta \mathrm{d} \tau=-\int_{\mathbf{R}^{2}}\left(\nabla^{f} A \cdot \partial_{\tau} B \cdot C+A \cdot \partial_{\tau} \nabla^{f} B \cdot C\right) \mathrm{d} \eta \mathrm{d} \tau .
$$

\section{References}

[1] Ambrosio, L., F. Serra Cassano, and D. Vittone: Intrinsic regular hypersurfaces in Heisenberg groups. - J. Geom. Anal. 16:2, 2006, 187-232.

[2] Barone Adesi, V., F. Serra Cassano, and D. Vittone: The Bernstein problem for intrinsic graphs in Heisenberg groups and calibrations. - Calc. Var. Partial Differential Equations 30:1, 2007, 17-49. 
[3] Capogna, L., D. Danielli, S. D. Pauls, and J. T. Tyson: An introduction to the Heisenberg group and the sub-Riemannian isoperimetric problem. - Progr. Math. 259, Birkhäuser Verlag, Basel, 2007.

[4] Danielli, D., N. Garofalo, D. M. Nhieu, and S. D. Pauls: Instability of graphical strips and a positive answer to the Bernstein problem in the Heisenberg group $\mathbf{H}^{1}$. - J. Differential Geom. 81:2, 2009, 251-295.

[5] Fogagnolo, M., R. Monti, and D. Vittone: Variation formulas for $H$-rectifiable sets. Ann. Acad. Sci. Fenn. Math. 42:1, 2017, 239-256.

[6] Franchi, B., R. Serapioni, and F. Serra Cassano: Rectifiability and perimeter in the Heisenberg group. - Math. Ann. 321:3, 2001, 479-531.

[7] Franchi, B., R. Serapioni, and F. Serra Cassano: Regular submanifolds, graphs and area formula in Heisenberg groups. - Adv. Math. 211:1, 2007, 152-203.

[8] Galli, M.: First and second variation formulae for the sub-Riemannian area in threedimensional pseudo-Hermitian manifolds. - Calc. Var. Partial Differential Equations 47:1-2, 2013, 117-157.

[9] Galli, M., and M. RitorÉ: Area-stationary and stable surfaces of class $C^{1}$ in the subRiemannian Heisenberg group $\mathbf{H}^{1}$. - Adv. Math. 285, 2015, 737-765.

[10] Garofalo, N., and D.-M. Nhieu: Isoperimetric and Sobolev inequalities for CarnotCarathéodory spaces and the existence of minimal surfaces. - Comm. Pure Appl. Math. 49:10, 1996, 1081-1144.

[11] Kirchheim, B., and F. Serra Cassano: Rectifiability and parameterization of intrinsic regular surfaces in the Heisenberg group. - Ann. Sc. Norm. Super. Pisa Cl. Sci. (5) 3:4, 2004, $871-896$.

[12] Korányi, A., and H. M. Reimann: Foundations for the theory of quasiconformal mappings on the Heisenberg group. - Adv. Math. 111:1, 1995, 1-87.

[13] Le Donne, E.: A primer on Carnot groups: homogenous groups, CC spaces, and regularity of their isometries. - arXiv:1604.08579, 2016.

[14] Le Donne, E., and S. Nicolussi Golo: Regularity properties of spheres in homogeneous groups. - Trans. Amer. Math. Soc., electronically published on September 7, 2017, DOI: https://doi.org/10.1090/tran/7038 (to appear in print).

[15] Montefalcone, F., and F. Serra Cassano: Intrinsic variational formulae for sets of finite $H$-perimeter in 2-step Carnot groups. - Work in progress, 2015.

[16] Monti, R.: Isoperimetric problem and minimal surfaces in the Heisenberg group. - In: Geometric measure theory and real analysis 17, CRM Series, Ed. Norm. Pisa, 2014, 57-129.

[17] Monti, R., and F. Serra Cassano, and D. Vittone: A negative answer to the Bernstein problem for intrinsic graphs in the Heisenberg group. - Boll. Unione Mat. Ital. (9) 1:3, 2008, 709-727.

[18] Serra Cassano, F.: Some topics of geometric measure theory in Carnot groups. - In: Geometry, analysis and dynamics on sub-Riemannian manifolds. Vol. 1, EMS Ser. Lect. Math., Eur. Math. Soc., Zürich, 2016, 1-121. 